\title{
On the Creation and Evolution of Small-Scale Low-Level Vorticity Anomalies during Tropical Cyclogenesis
}

\author{
WARren P. SMith AND Melville E. Nicholls \\ Cooperative Institute for Research in Environmental Sciences, Department of Atmospheric and Oceanic \\ Sciences, University of Colorado Boulder, Boulder, Colorado
}

(Manuscript received 4 April 2018, in final form 16 May 2019)

\begin{abstract}
Recent numerical modeling and observational studies indicate the importance of vortical hot towers (VHTs) in the transformation of a tropical disturbance to a tropical depression. It has recently been recognized that convective-scale downdraft outflows that form within VHTs also preferentially develop positive vertical vorticity around their edges, which is considerably larger in magnitude than ambient values. During a numerical simulation of tropical cyclogenesis it is found that particularly strong low-level convectively induced vorticity anomalies (LCVAs) occasionally form as convection acts on the enhanced vorticity at the edges of cold pools. These features cycle about the larger-scale circulation and are associated with a coincident pressure depression and low-level wind intensification. The LCVAs studied are considerably deeper than the vorticity produced at the edges of VHT cold pool outflows, and their evolution is associated with persistent convection and vortex merger events that act to sustain them. Herein, we highlight the formation and evolution of two representative LCVAs and discuss the environmental parameters that eventually become favorable for one LCVA to reach the center of a larger-scale circulation as tropical cyclogenesis occurs.
\end{abstract}

\section{Introduction}

Hurricanes are powerful cyclonically rotating systems that lead to widespread destruction in coastal areas every year. An important early stage in the formation of a hurricane is the transformation from a tropical disturbance to a tropical depression, a process known as tropical cyclogenesis (TCG). TCG is difficult to predict because it is governed by many simultaneous kinematic and thermodynamic processes occurring at different spatial and temporal scales that are not well understood. Such processes include the development of midlevel vortices (MLVs; horizontal scale of order $100 \mathrm{~km}$ ) that may persist for several days and rotating convective towers (horizontal scale of order $10 \mathrm{~km}$ ) that may persist for a few hours. A possible path to TCG has recently been proposed, which states that after a MLV forms in a tropical disturbance, a much smaller convective-scale vortex may arrive in its center that subsequently develops rapidly into a tropical cyclone (TC; e.g., Nolan 2007; Nicholls and Montgomery 2013). The current

Corresponding author: Warren P. Smith, warren.p.smith@ colorado.edu work investigates the origin of such small vortices and what conditions might be favorable for one to propagate to the center of the larger-scale circulation.

MLVs have often been observed and modeled as a precursor to TCG (e.g., Bister and Emanuel 1997; Raymond and López Carrillo 2011; Davis and Ahijevych 2012; Kutty and Gohil 2017; Nicholls et al. 2018), and their formation has been associated with the sudden appearance of a small surface-concentrated vortex (SSCV) in its center that then becomes the low-level core of an intensifying TC (Nolan 2007). In the simulations performed by Nicholls and Montgomery (2013) it was noted that these smaller vortices typically originate tens of kilometers from the larger-scale center rather than forming there. Why these convective-scale vortices behave as they do has prompted calls for more modeling studies in order to further our understanding of how they might contribute to TCG (Fang and Zhang 2011).

Beneath a MLV, rotating convection has been shown to be an important contributor to TCG (e.g., Hendricks et al. 2004; Montgomery et al. 2006; Fang and Zhang 2011). The term "vortical hot tower" (VHT) has been coined to describe convective-scale rotating cells that span the depth of the troposphere and last for approximately 
$1 \mathrm{~h}$ as they propagate within a larger-scale circulation prior to TCG (Hendricks et al. 2004). VHTs provide a protected environment for efficient conversion of latent heat into rotational momentum and can merge with other VHTs to become stronger (Montgomery et al. 2006). A larger-scale circulation can protect a developing disturbance from the lateral entrainment of dry air that would otherwise inhibit moist convection from occurring (Dunkerton et al. 2009; Raymond and López Carrillo 2011). This VHT "pathway" to TCG is somewhat distinct from the aforementioned MLV hypothesis because it relies on rotating convective-scale structures that provide enhanced vertical vorticity for the eventual formation of a larger-scale tropical depression. A modeling study by Nicholls and Montgomery (2013) showed that both the VHT mechanism (Hendricks et al. 2004; Montgomery et al. 2006) and the MLV with an SSCV mechanism (Nolan 2007) manifested in the same numerical modeling framework depending on the initial conditions employed.

Like other forms of tropical deep convection, VHTs produce cold pools from precipitating downdrafts that spread at the surface (e.g., Eastin et al. 2012), and discrete convective towers often form downshear of such cold pools (Davis 2015). Cold pools produced in this way tend to be shallow (with depths of just a few hundred meters) and often generate positive vorticity at the edge of their spreading outflow that could promote the development of discrete vortices if acted upon by additional convection (Nicholls and Montgomery 2013). The term "convectively induced vorticity anomalies" (CVAs) has been given to tropical rotating vortices of different sizes and origins, which are often building blocks for larger scales of rotation (Fang and Zhang 2011). We choose to add a "low-level" modifier to this acronym by distinguishing smaller vortices at the edges of cold pools from VHTs, as we hypothesize they can contribute to TCG in fundamentally different ways.

The present study focuses on the life cycle of strong lowlevel convectively induced vorticity anomalies (LCVAs) that form sporadically and propagate cyclonically for up to $8 \mathrm{~h}$ in a numerical simulation prior to TCG. We show that eventually the background conditions of the tropical disturbance become favorable for one LCVA to move into the center and become the low-level core of an intensifying $\mathrm{TC}$, in a manner analogous to the "appearance" of an SSCV in the work of Nolan (2007). This study focuses on exploring two such LCVAs in a numerical simulation: one that dissipates about $8 \mathrm{~h}$ after its formation (which we term the "early LCVA") and one that eventually moves into the center of the larger-scale circulation as TCG occurs (which we term the "genesis LCVA"). A vorticity budget is performed on the formation of an LCVA to show that they form as convection acts upon enhanced regions of vorticity at the edges of cold pools. Insights are made into the presence of shear and curvature vorticity in the formation of LCVAs and the presence of vortex merger events is examined during their life cycle. In doing so, the present understanding of the mechanisms by which these vortices form is improved and insight is provided for the background conditions that allow for increased longevity of LCVAs as time progresses.

The remainder of this paper is organized as follows. The next section describes the numerical model setup. Section 3 discusses the formation, development, and dissipation mechanisms of LCVAs. Section 4 discusses the concept of shear and curvature vorticity, and section 5 tests the hypothesis that vortex mergers are occurring that strengthen LCVAs. Discussion and conclusions are given in sections 6 and 7. Appendixes pertaining to cyclostrophic and hydrostatic balances, the presence of surface-based convective available potential energy (SBCAPE) in LCVAs, and a friction estimation for the curvature vorticity budget are also included.

\section{Numerical model and methods}

Because TCG typically occurs in areas that are far from landmasses, much of the research in the field is performed with the aid of numerical modeling (Pielke and Pielke 1997). The environments that TCs form in are often complex, and for simplicity many numerical modeling studies have examined development in idealized conditions. Probably the most canonical model configuration is one without any ambient winds, that has a favorable thermodynamic environment with a conditionally unstable troposphere overlying a warm ocean surface, and that is initialized with a weak mesoscale vortex [such as that used in Nicholls and Montgomery (2013)].

The Regional Atmospheric Modeling System (RAMS), version 4.3 (Pielke et al. 1992; Cotton et al. 2003), is employed for the simulation presented in this study. RAMS is a three-dimensional nonhydrostatic numerical modeling system that uses time-dependent equations for velocity, nondimensional pressure perturbation, ice-liquid water potential temperature (Tripoli and Cotton 1981), total water mixing ratio, and cloud microphysics. The microphysics parameterization explicitly calculates rain, cloud water, pristine ice, snow, aggregates, graupel, and hail mixing ratios (Walko et al. 1995; Meyers et al. 1997). The simulation analyzed in this study is the same as that discussed in Nicholls et al. (2018), who instead analyzed the formation and evolution of a MLV. The reader is directed to their Fig. 1 for a broad overview of how this simulation evolves. Below we restate how this simulation is initialized. 
The analyzed simulation is initiated with the hurricane sounding from Jordan (1958), which is slightly dried at low levels (as discussed in Nicholls and Montgomery 2013). Potential temperature perturbations in this work are calculated as the deviation from this horizontally homogeneous initial sounding. The simulation is initialized with a broad vortex with peak wind speeds of $8 \mathrm{~m} \mathrm{~s}^{-1}$ at a height of $4 \mathrm{~km}$ and a radius of maximum winds (RMW) of $75 \mathrm{~km}$ (not shown). In all of this paper's figures, the point $(0,0) \mathrm{km}$ is defined as the center of this larger-scale vortex with positive (negative) values of $x$ and $y$ lying to the east and north (west and south), respectively.

The sea surface temperature (SST) is set as a constant $28^{\circ} \mathrm{C}$ in this simulation, which is a typical value in the tropics during hurricane season and is favorable for TCG (Gray 1968). For simplicity, the Coriolis force is approximated using an $f$ plane at a latitude of $15^{\circ} \mathrm{N}$. The model domain is initially devoid of any liquid or solid water, and no longwave or shortwave radiation schemes are employed. Subgrid-scale turbulence is parameterized according to deformation- $K$ closure found in Smagorinsky (1963) with stability modifications from Lilly (1962) and Hill (1974).

The simulation contains three concentric grids that have horizontal grid spacings of $12 \mathrm{~km}(170 \times 170$ points $), 3 \mathrm{~km}$ $(202 \times 202$ points $)$, and $1 \mathrm{~km}(302 \times 302$ points $)$. All three grids have 47 vertical levels that are vertically stretched from the lowest model level at $29.5 \mathrm{~m}$ above ground level (AGL) to the top at approximately $23 \mathrm{~km}$ AGL. A Rayleigh damping layer is added to suppress gravity waves from reaching the model top, which begins at a height of $15.2 \mathrm{~km}$ AGL. Only output from the finest (1-km spacing) grid is considered in the following analysis.

The vertical component of relative vorticity (hereafter just "vorticity") and horizontal divergence (hereafter just "divergence") are computed, respectively, by

$$
\begin{gathered}
\zeta=(\nabla \times \mathbf{u}) \cdot \hat{\mathbf{k}}=\frac{\partial v}{\partial x}-\frac{\partial u}{\partial y}, \\
\delta_{h}=\nabla_{h} \cdot \mathbf{u}_{h}=\frac{\partial u}{\partial x}+\frac{\partial v}{\partial y} .
\end{gathered}
$$

In the next section we perform a vorticity analysis on the formation of the early LCVA using the material form of the vertical vorticity budget equation with friction neglected:

$$
\begin{aligned}
\frac{\partial \zeta}{\partial t}= & -(\mathbf{u} \cdot \nabla) \zeta-\delta_{h}(f+\zeta)-\left(\frac{\partial w}{\partial x} \frac{\partial v}{\partial z}-\frac{\partial w}{\partial y} \frac{\partial u}{\partial z}\right) \\
& +\frac{1}{\rho^{2}}\left(\frac{\partial \rho}{\partial x} \frac{\partial p}{\partial y}-\frac{\partial \rho}{\partial y} \frac{\partial p}{\partial x}\right)
\end{aligned}
$$

where $\mathbf{u}=\langle u, v, w\rangle$ is the three-dimensional wind vector where $u, v$, and $w$ are the zonal, meridional, and vertical components of the wind, respectively; $f=2 \Omega \sin (\phi)$ is the Coriolis parameter for Earth's angular velocity $\Omega=7.29 \times 10^{-5} \mathrm{~s}^{-1}$ and latitude $\phi ; \rho$ is the air density; and $p$ is the pressure.

The term on the left-hand side of Eq. (3) is the Eulerian derivative of vorticity with respect to time. The first term on the right-hand side represents advection and the second term is the stretching term, which describes vorticity changes from horizontal convergence and divergence. The third term on the right-hand side of Eq. (3) is the tilting term, which describes the conversion of horizontal vorticity into vertical vorticity through horizontal gradients of vertical velocity. The final term is the baroclinic (or "solenoid") term, which describes the production of vorticity through a comparison of horizontal gradients in density and pressure.

Because RAMS uses Arakawa-C grid staggering (Arakawa and Lamb 1977), wind fields are first linearly interpolated onto the same points as the thermodynamic variables before computations are performed. Partial derivatives are then calculated at each point using the adjacent grid points in the appropriate dimension.

\section{Analysis of LCVAs}

In this section, we describe the formation and intensification of two distinct LCVAs that propagate through the domain prior to TCG. These LCVAs are fundamentally different than VHTs in that they have maximum vorticity near the surface rather than at midlevels, which was suggested by Fig. 5 of Hendricks et al. (2004). The first LCVA analyzed is termed the "early LCVA" because it forms and dissipates long before TCG occurs. The other LCVA is termed the "genesis LCVA" because it does eventually reach the center of the largerscale circulation, an event that coincides with the timing of TCG. The spatial propagation and intensity evolution of both these LCVAs is shown in Fig. 1. It is important to note that these two LCVAs are not the only two that exist in the simulation, but these two are selected as representative in order to simplify the forthcoming analysis. Analyzing how these LCVAs form and how they interact with their surrounding environments gives clues as to how it is eventually conducive for an LCVA to reach the center of the larger-scale circulation.

\section{a. The early LCVA}

Figure 1a shows the early LCVA's horizontal track and a time series of its intensity from 43- to 52-h run time. The tracks in this figure are produced by subjective selection of the vorticity maximum in the LCVA. 

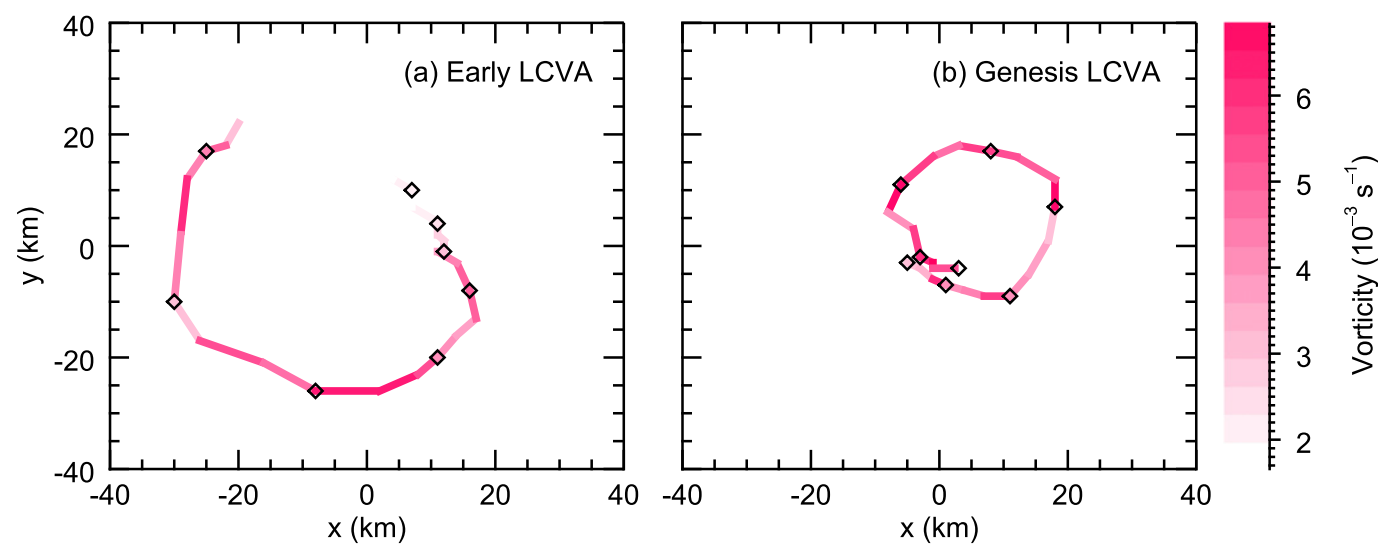

FIG. 1. Horizontal motion of the (a) early LCVA from $43 \mathrm{~h} 20 \mathrm{~min}$ to $51 \mathrm{~h} 20 \mathrm{~min}$ run time and (b) the genesis LCVA from 83- to 90-h run time. Each line segment represents motion over a 20-min period, and the color of each segment shows the average value of $\zeta\left(10^{-3} \mathrm{~s}^{-1}\right)$ calculated for a $2 \mathrm{~km} \times 2 \mathrm{~km}$ grid box centered on the LCVA at the lowest model level $(z=29.5 \mathrm{~m} \mathrm{AGL})$. Black diamond symbols represent the location of the LCVA at every hour (on the hour) to give information about propagation speed. Tracks are defined by a subjective selection method of the low-level vorticity maximum at each time step.

LCVAs move counterclockwise along with the cyclonic flow of the larger-scale vortex (discussed in section 2). The early LCVA first is distinguishable as a quasicircular region of enhanced vorticity at the edge of a cold pool, shown in Fig. 2a. Figure 2d shows the location of the cold pool to its southeast. This cold pool is roughly $300 \mathrm{~m}$ deep, which is a representative depth for this simulation (not shown). The strongest convective cold pools in this simulation have potential temperature depressions of about $4 \mathrm{~K}$, consistent with other observational and modeling studies in tropical environments (Eastin et al. 2012; Drager and van den Heever 2017).

In Fig. 2, the edge of the cold pool is marked by enhanced vorticity (Fig. 2a), enhanced convergence (negative divergence in Fig. 2b) and therefore an enhanced stretching term in Fig. $2 \mathrm{~g}$. This mechanism tends to create positive vorticity at the edges of cold pools because the ambient vorticity inside the RMW is generally positive. The vertical velocity field in Fig. $2 \mathrm{c}$ indicates that deep convection is associated with the LCVA at this time. The convective column is tilted toward the south (by comparison of the filled and black contour fields) indicating that the convective downdraft does not impede convection from continuing to act on the LCVA. By comparing all terms contributing to vorticity changes (Fig. 2e-i), it can be seen that the stretching term and horizontal advection are the dominant terms in Eq. (3). Convection acts to stretch even more vorticity in the column as time progresses. This analysis suggests that shallow vorticity at the edges of cold pools can be stretched to produce vortices by updrafts in convective cells and that these vortices may become more intense from the vorticity present in their surroundings.
Figure 3 shows horizontal and vertical cross sections of the early LCVA at $46 \mathrm{~h}$. The early LCVA is being acted on by convection that causes it to grow from shallow vorticity at the edge of a cold pool (depth of about $500 \mathrm{~m}$ ) to about $6 \mathrm{~km}$ deep (Fig. 3a). This vorticity is on the order of $10^{-2} \mathrm{~s}^{-1}$, which is considerably higher than background values, an observation consistent with the discussion of intense rotating convection in Davis (2015). In the horizontal, the LCVA occupies approximately $10 \mathrm{~km} \times 5 \mathrm{~km}$ near the surface (Fig. $3 \mathrm{c}$ ). There are no significant cold pools visible near the LCVA at this time (Fig. 3d), but deep convection is present on its west side as evidenced by the vertical cross section of total hydrometeor mixing ratio (Fig. 3b).

Figure 4 shows the same cross sections as in Fig. 3, except $3 \mathrm{~h} 40 \mathrm{~min}$ later at $49 \mathrm{~h} 40 \mathrm{~min}$. The early LCVA has weakened remarkably and now only extends to about $2 \mathrm{~km}$ AGL (Fig. 4a). Convection is absent near the LCVA (Fig. 4b), which prevents it from retaining its former depth, and its vorticity is drawing closer to ambient values of about $10^{-3} \mathrm{~s}^{-1}$ (Fig. 4c). Two new cold pools are now evident, centered at approximately (37, 20) $\mathrm{km}$ and $(-25,-17) \mathrm{km}$ (Fig. 4d). Both cold pools contain noteworthy enhancements in vorticity nearby, which are now stronger than the early LCVA. LCVAs continue to develop and dissipate in a similar fashion as the simulation progresses, until the background conditions eventually become conducive for the genesis LCVA to reach the center about $40 \mathrm{~h}$ later.

\section{b. The genesis LCVA}

We now move ahead in time to examine another LCVA that eventually reaches the center of the domain 

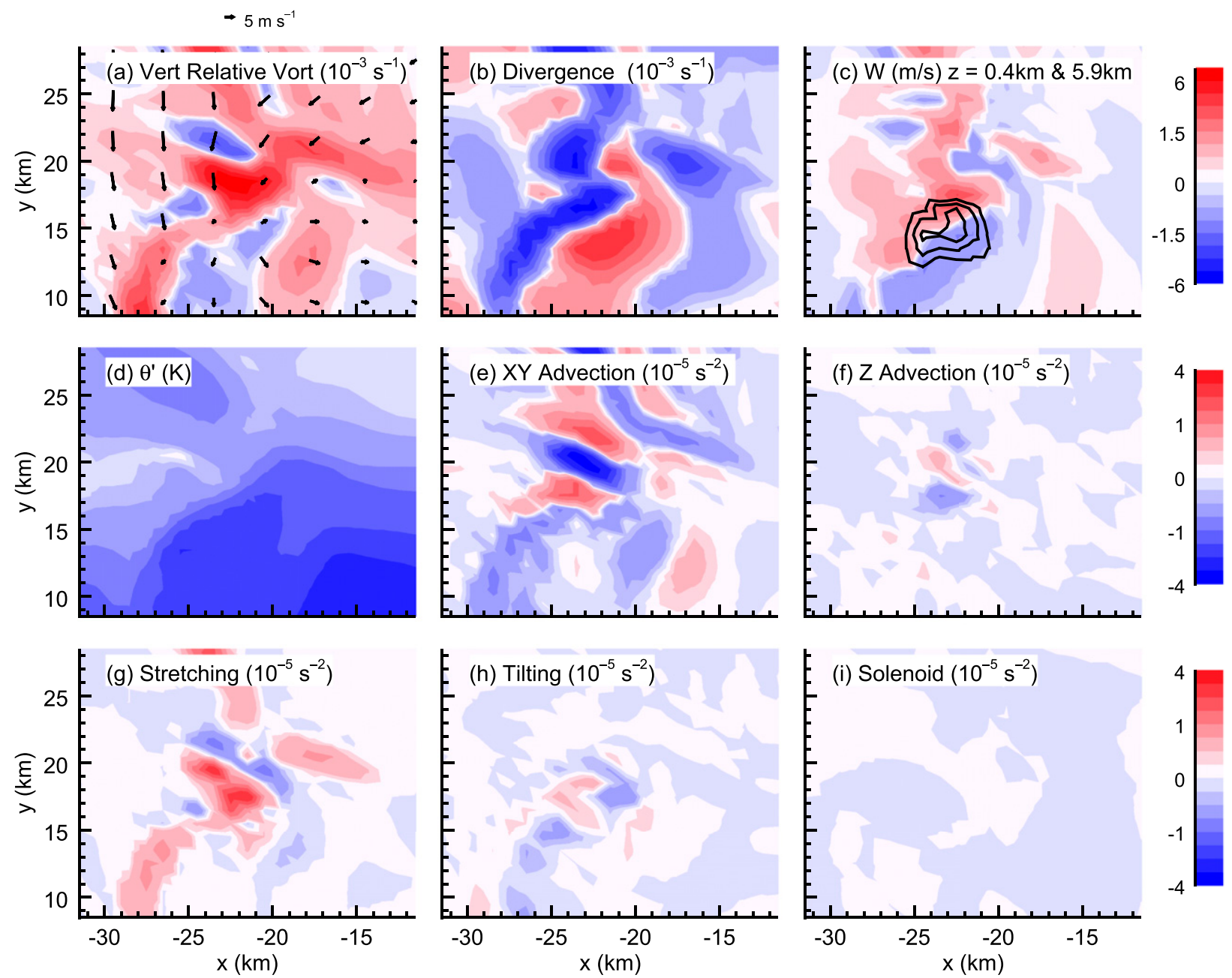

FIG. 2. Horizontal cross sections of (a) vertical vorticity with horizontal wind vectors, (b) horizontal divergence, (c) vertical velocity, (d) perturbation potential temperature, and the (e) horizontal advection, (f) vertical advection, (g) stretching, (h) tilting, and (i) solenoid terms from Eq. (3) at a model run time of $43 \mathrm{~h} 40 \mathrm{~min}$. All fields are taken at the lowest model level $(z=29.5 \mathrm{~m} \mathrm{AGL})$ except (c), which shows vertical velocity at $z=0.4$ (shading) and $5.9 \mathrm{~km} \mathrm{AGL} \mathrm{(black} \mathrm{contours} \mathrm{at} 4,8$, and $12 \mathrm{~m} \mathrm{~s}^{-1}$ ). Vorticity and divergence are scaled by a factor of $10^{3} \mathrm{~s}^{-1}$ and all terms from Eq. (3) are scaled by a factor of $10^{5} \mathrm{~s}^{-1}$.

and intensifies, which we term the "genesis LCVA." Figure $1 \mathrm{~b}$ shows the track and intensity of the genesis LCVA from $83 \mathrm{~h}$ through TCG at $90 \mathrm{~h}$. Figure 5 shows its birth, illustrated by low-level cross sections of vorticity and perturbation potential temperature. At about $82 \mathrm{~h}$, two cold pools form very near to each other (Fig. 5b). As these two cold pools mature (Fig. 5d) they create particularly strong vorticity along their interface brought about by stretching from opposing outflow directions (Fig. 5c, and as in Fig. 2 for the early LCVA). This interaction spurs deep convection, which concentrates vorticity into a column and leads to the formation of the genesis LCVA (Fig. 5e).

Figure 6 shows vertical cross sections of vertical velocity and vorticity at various times through the center of the genesis LCVA, illustrating its development and intensification. Figure 6a shows a shallow region of enhanced vorticity that is present at the edge of a convective cold pool (similar to that shown in Fig. 2 for the early LCVA). To its east in Figs. 6a and 6b, the remnants of the VHT that created the cold pool are still visible. This enhanced vorticity suddenly deepens to about $6 \mathrm{~km}$ AGL when acted upon by convection 40 min later (Figs. 6c,d). Convection continues to act on the LCVA throughout its life cycle (Figs. 6e,f) until finally it is able to reach the center of the larger-scale circulation (Figs. 6g,h), when TCG occurs (as evidenced by a rapid climb in tangential winds and rapid decrease of surface pressure beginning at this time). After this time, the LCVA continues to intensify and the pressure begins to drop as the low-level vortex transforms into a tropical depression. The rapid regeneration of SBCAPE favors 

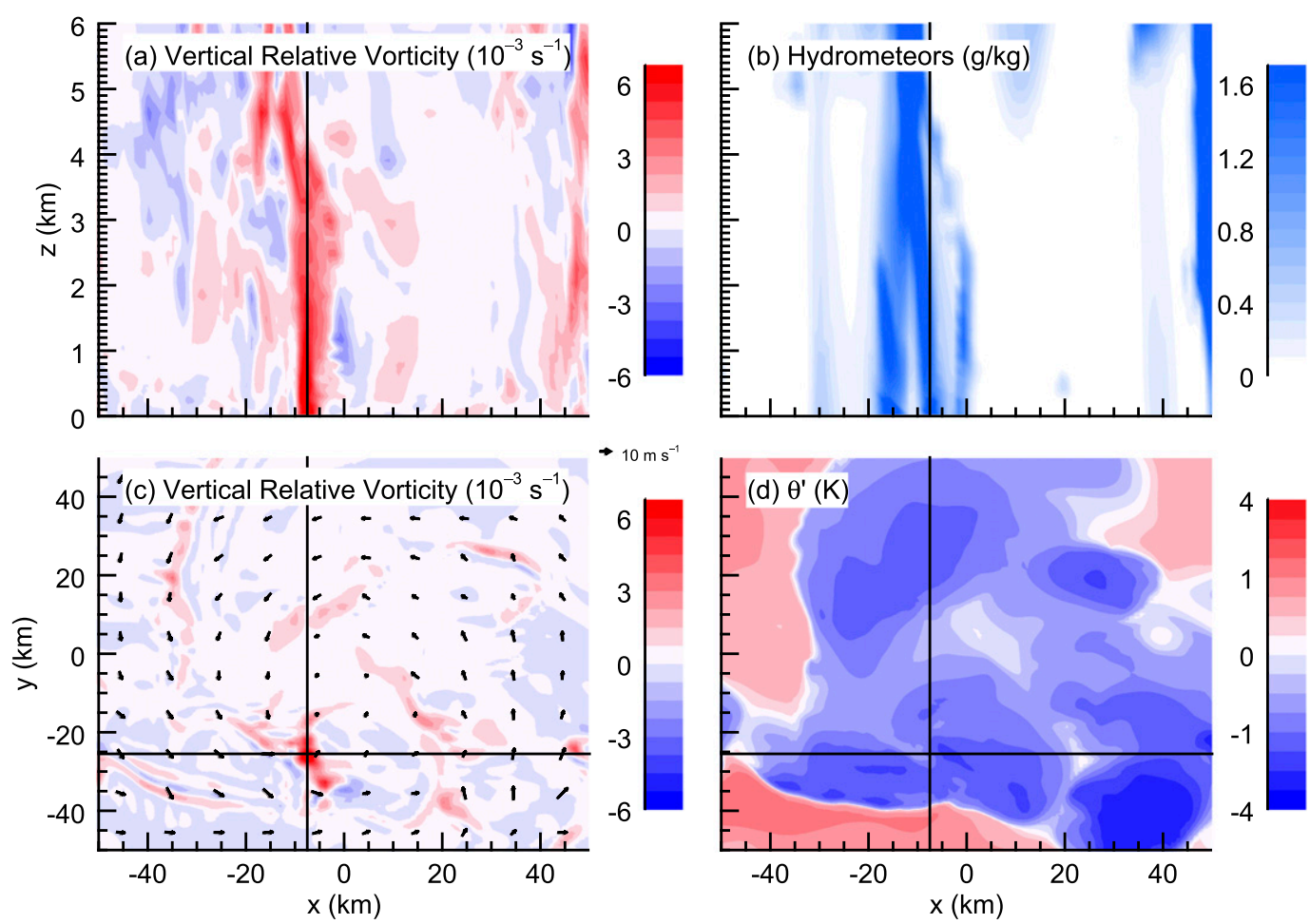

FIG. 3. (top) Vertical cross sections of (a) vertical vorticity and (b) total liquid and ice hydrometeor mixing ratio and (bottom) horizontal cross sections of (c) vertical vorticity with horizontal wind vectors and (d) perturbation potential temperature taken at the lowest model level $(z=29.5 \mathrm{~m} \mathrm{AGL})$ at a model run time of $46 \mathrm{~h}$. Vertical cross sections are taken through the horizontal lines in the horizontal cross sections. Vertical lines are in the same location in all plots for reference only. Vorticity fields are scaled by a factor of $10^{3} \mathrm{~s}^{-1}$.

persistent convection throughout the genesis LCVA's life cycle (see appendix B).

The bend in the convective column in Figs. $6 \mathrm{~d}$ and $6 \mathrm{f}$ is the result of local vertical wind shear from a strong MLV that develops in this simulation (Nicholls et al. 2018). This is illustrated more explicitly in Fig. 7, which shows vertical cross sections at $86 \mathrm{~h} 20 \mathrm{~min}$. A strong MLV (between 4 and $6 \mathrm{~km}$ AGL) can be seen in Fig. $7 \mathrm{~b}$ that causes some local vertical wind shear, thus tilting the column of vorticity (Fig. 7a). This vertical shear promotes updrafts and downdrafts to not be collocated (Figs. 7c,d), which may increase the average lifetime of a convective tower. Therefore we hypothesize that the strengthening and contraction of the MLV throughout the simulation is conducive to longer-lasting and stronger LCVAs by promoting vertically tilted convective columns that are sustained longer. This same mechanism was briefly mentioned in appendix A of Nicholls and Montgomery (2013).

Throughout the evolution of both the early and genesis LCVAs, a spatially coincident region of lowered pressure (maximum depression around $2 \mathrm{hPa}$ from the surroundings) exists and synchronously strengthens and weakens with the LCVAs. This pressure drop for the genesis LCVA is illustrated in Fig. 8, which shows horizontal cross sections of vorticity and pressure perturbation at two times $20 \mathrm{~min}$ apart. Between the two time steps, convection acts on the genesis LCVA, which causes it to intensify at the surface (Figs. 8a,c). Consequently, the pressure depression increases slightly and its horizontal extent is reduced to match that of the genesis LCVA (Fig. 8b,d). This same process can be seen in the early LCVA as well, but is not shown in the interest of brevity. Pressure depressions are persistent features in LCVAs and are a likely catalyst for promoting TCG when an LCVA reaches the center, as this begins the fall of the central surface pressure.

Figure 9 shows the same fields as in Fig. 3, except at a model run time of $90 \mathrm{~h}$, when TCG occurs. At this time the genesis LCVA is very strong and reaches the center of the larger-scale circulation. The vorticity maximum is found along the edge of a central cold pool that likely forms from convective precipitation falling out downshear of the LCVA (see Fig. 7). Vorticity at the edge of this cold pool may also contribute to the strengthening of the LCVA as it moves toward the center of the larger-scale circulation. At this time the genesis LCVA is 

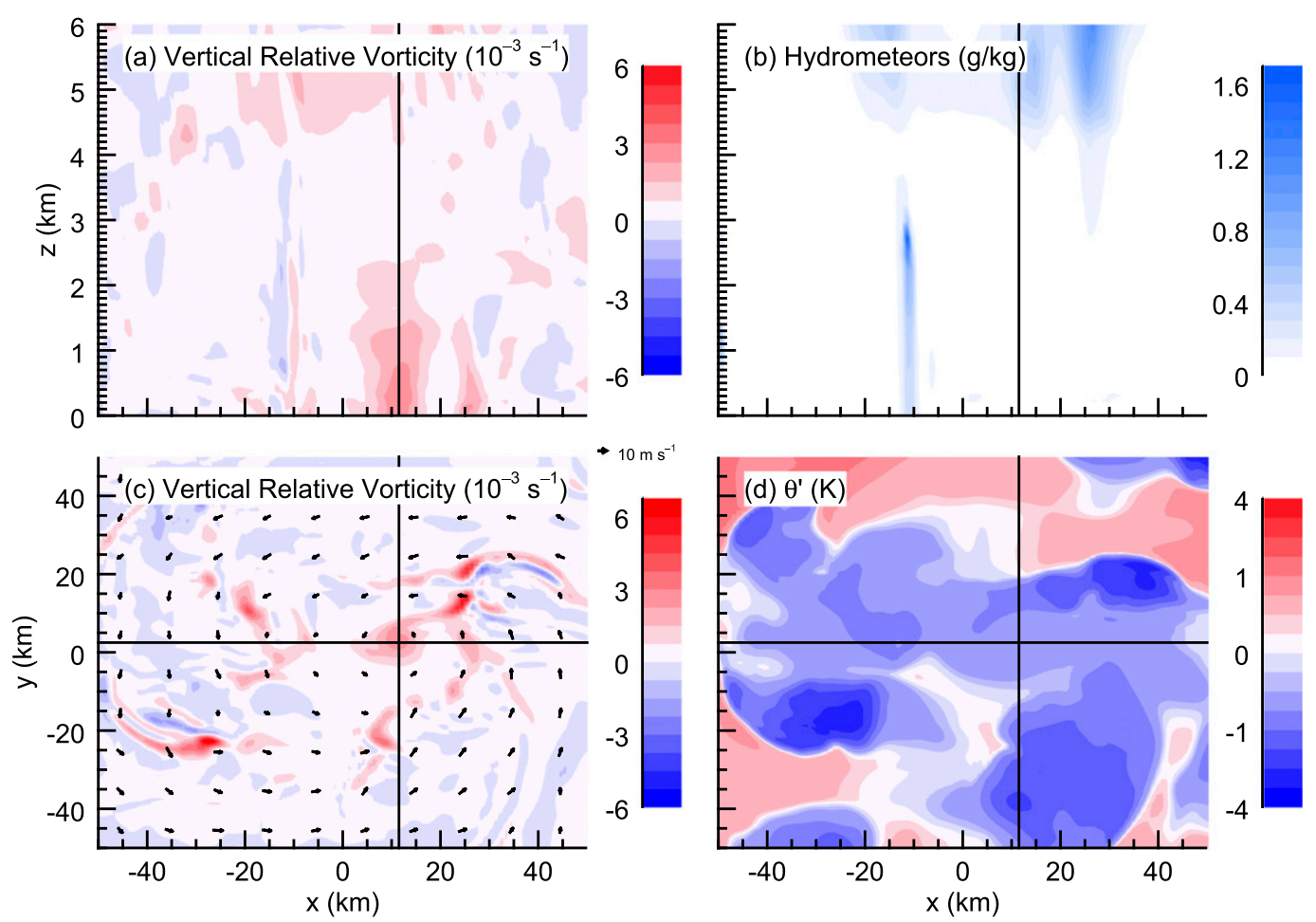

FIG. 4. As in Fig. 3, but at a model run time of $49 \mathrm{~h} 40 \mathrm{~min}$.

now analogous to the SSCV from Nolan (2007) that signals TCG is occurring.

We have shown that LCVAs in this simulation first appear as shallow vorticity signatures at the edges of cold pools that come about from the horizontal wind shift between their convective outflow and the ambient vortex (Figs. 2 and 5). The subsequent presence of convection allows for the concentration of vorticity into a more circular column. These two states represent "shear" and "curvature" vorticity, respectively (Saucier 1955). In the next section, we break down the vorticity field into these components and analyze how curvature vorticity in LCVAs can form from shear vorticity at the edges of cold pools.

\section{Shear and curvature vorticity}

In this section, we examine the formation of shear vorticity at the edges of cold pools and its subsequent conversion to curvature vorticity as LCVAs form. In particular we focus on the formation of the genesis LCVA between 83 and $84 \mathrm{~h}$. We consider shear and curvature vorticity in natural coordinates defined by $-\delta V / \delta n$ and $V \delta \alpha / \delta s$, respectively, for wind speed $V$ and angle of the $s$ axis with respect to the $x$ axis $\alpha$. Partial derivatives with respect to $s$ and $n$ are calculated along the local wind direction and normal to it, respectively, by the definition of a natural coordinate system (e.g., Holton 1992, 61-62). Shear and curvature vorticity are not frame invariant; however, the system motion provides a suitable frame for our computations.

We consider the curvature vorticity budget equation employed by Schenkel (2009) originally adapted from Bell and Keyser (1993):

$$
\begin{aligned}
\frac{d}{d t}\left(V \frac{\delta \alpha}{\delta s}\right)= & -V \frac{\partial f}{\partial s}-\frac{\delta V}{\delta s} \frac{d \alpha}{d t}-\frac{\delta}{\delta n}\left(\frac{\delta \Phi}{\delta s}\right) \\
& -\left(f+V \frac{\delta \alpha}{\delta s}\right) \nabla_{p} \cdot \mathbf{V}-V \frac{\delta \omega}{\delta s} \frac{\partial \alpha}{\partial p}+F_{\zeta_{c}}
\end{aligned}
$$

The left-hand side of Eq. (4) is the Lagrangian time derivative of curvature vorticity. On the right-hand side, the first term is the change in curvature vorticity from advection in a planetary vorticity gradient. This term is zero for our simulation because of the $f$-plane approximation. The second and third terms on the right-hand side are the shear to curvature vorticity conversion terms, where $\Phi$ is geopotential. These terms appear exactly opposite in the shear vorticity budget equation (not shown), so these terms represent curvature vorticity being produced exclusively at the expense of shear vorticity. The two terms are summed together for the following analysis. The fourth and fifth terms on the 

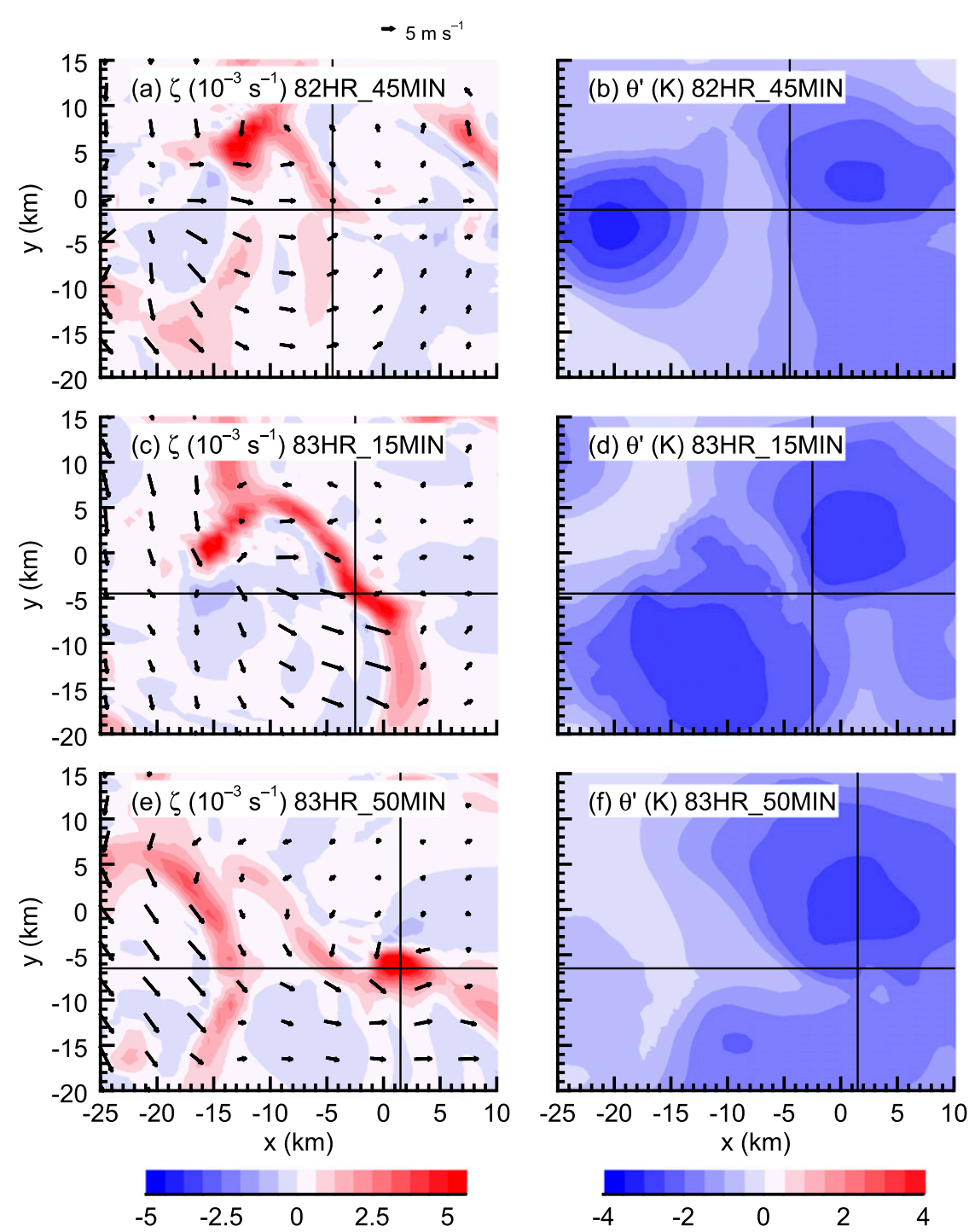

FIG. 5. Horizontal cross sections of (left) vertical vorticity with horizontal wind vectors and (right) perturbation potential temperature taken at the lowest model level ( $z=29.5 \mathrm{~m}$ AGL) at (a),(b) $82 \mathrm{~h} 40 \mathrm{~min}$, (c),(d) $83 \mathrm{~h} 15 \mathrm{~min}$, and (e),(f) $83 \mathrm{~h} 50 \mathrm{~min}$. The crosshairs in each plot mark the genesis LCVA. Vorticity fields are scaled by a factor of $10^{3} \mathrm{~s}^{-1}$.

right-hand side represent stretching and tilting of curvature vorticity, respectively, where $\nabla_{p}$ is the gradient operator in natural coordinates, and $\omega$ is the vertical velocity in pressure coordinates (O'Brien 1970). The final term on the right-hand side represents our estimate for friction; the exact formulation for this is provided in appendix C. Directional derivatives (denoted by $\delta$ ) are used in the equation to account for grid points in which a natural coordinate system is not defined in order to be consistent with Schenkel (2009), although there are no such points in our simulation. Prior to performing calculations, the horizontal wind, geopotential, and pressure fields are smoothed once with a 1-2-1 filter.
Figure 10 shows horizontal cross sections taken at a model run time of $83 \mathrm{~h} 22 \mathrm{~min}$. In this figure, shear and curvature vorticity as well as terms from Eq. (4) are smoothed once with a 1-2-1 filter after computations are performed to remove excess noise in the fields. Shown are the two adjacent cold pools responsible for the birth of the genesis LCVA (Fig. 10d; see Fig. 5) and an enhanced region of vorticity along the interface between them (Fig. 10a). A significant portion of this vorticity is shear vorticity (cf. Figs. 10b and 10e) brought about by the different wind directions in the two opposing convective outflows. Figure $10 \mathrm{~h}$ indicates that curvature vorticity is being created at the expense of shear 

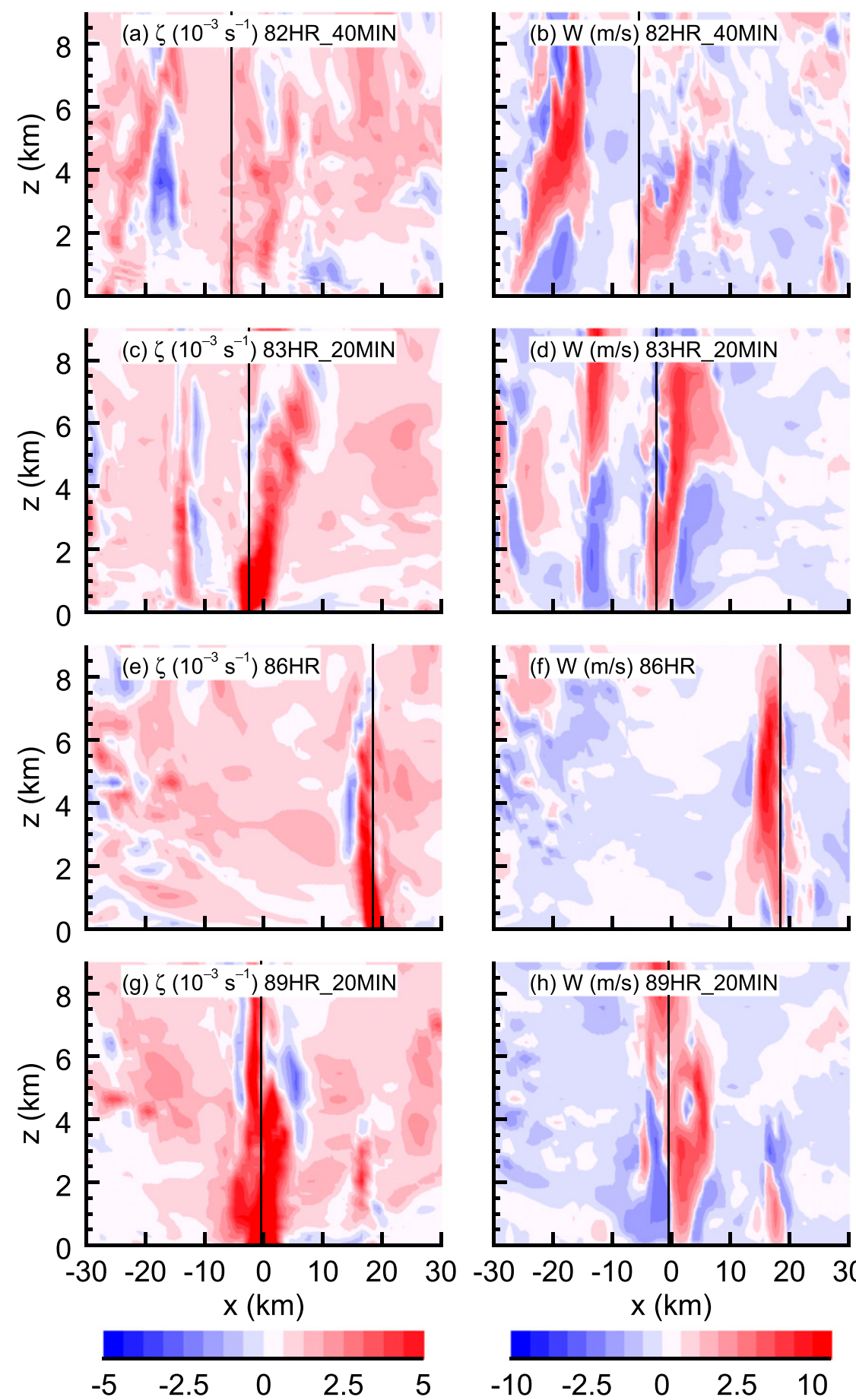

FIG. 6. Vertical cross sections of (left) vertical vorticity and (right) vertical velocity taken through the center of the genesis LCVA at (a),(b) $y=0.5 \mathrm{~km}$ at $82 \mathrm{~h} 40 \mathrm{~min}$, (c),(d) $y=-3.5 \mathrm{~km}$ at $83 \mathrm{~h}$ $20 \mathrm{~min}$, (e),(f) $y=7.5 \mathrm{~km}$ at $86 \mathrm{~h}$, and (g),(h) $y=-2.5 \mathrm{~km}$ at $88 \mathrm{~h} 40 \mathrm{~min}$. The vertical lines in each plot mark the center of the LCVA at the surface. Vorticity fields are scaled by a factor of $10^{3} \mathrm{~s}^{-1}$.

vorticity while Figs. $10 \mathrm{~g}$ and $10 \mathrm{i}$ indicate that the curvature vorticity present is beginning to intensify from stretching and tilting. Figure $10 \mathrm{f}$ shows the sum of these three lowest panels and the friction term (not shown) for comparison with the time derivative of curvature vorticity (Fig. 10c). While not perfect, these two panels show many similar features, indicating that there is reasonable closure of Eq. (4). Minor discrepancies may be due to our 

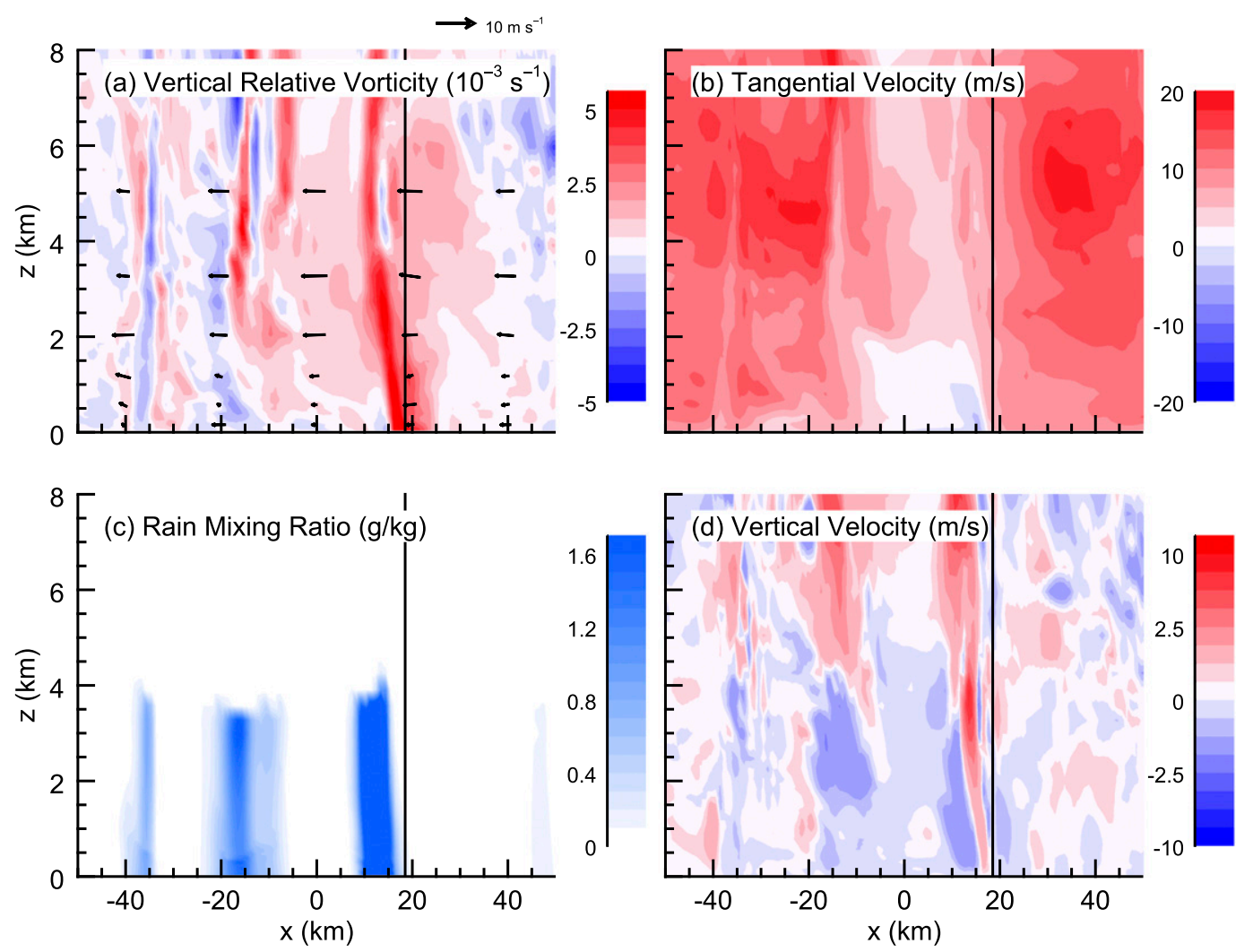

FIG. 7. Vertical cross sections of (a) vertical vorticity with zonal-vertical wind vectors, (b) tangential wind, (c) rain mixing ratio, and (d) vertical velocity taken at $y=12.5 \mathrm{~km}$ (through the center of the genesis LCVA) at a model run time of $86 \mathrm{~h} 20 \mathrm{~min}$. Tangential wind is calculated relative to the model center, with positive values indicating cyclonic motion. This means that values to the east (west) of the center are going into (out of) the page in (b). Vorticity is scaled by a factor of $10^{3} \mathrm{~s}^{-1}$.

estimation of friction, which is computed from model output rather than being output from the model directly (see appendix C). While shear vorticity conversion does not appear to dominate the production of curvature vorticity, its magnitude suggests that it is an important process to consider in the formation of this LCVA.

Figure 11 shows the same fields as Fig. 10, except $12 \mathrm{~min}$ later at a model run time of $83 \mathrm{~h} 34 \mathrm{~min}$. The genesis LCVA is now visible in Fig. 11a as a coherent vortex, which is shifted to the east because of both the background vortex and the cold pool outflow coming from the west (Fig. 10d and the vectors in Fig. 10a). Although some residual shear vorticity remains (Fig. 11e), more curvature vorticity is present than before (Fig. 11b), owing primarily to conversion from shear vorticity and the stretching of curvature vorticity (as argued in the previous paragraph). A comparison of the stretching and tilting terms (Figs. 11g,i) indicates that stretching is more important than tilting in continuing to generate curvature vorticity, which is consistent with the argument presented in section 3 (see Fig. 2). The stretching term now dominates the shear to curvature term, which is expected as the LCVA shifts to primarily curvature vorticity. Figures $11 \mathrm{c}$ and $11 \mathrm{f}$ still show a reasonable agreement between the sides of Eq. (4).

The preceding analysis is evidence that LCVAs can originate from convective outflows in the presence of ambient vorticity and convection. The enhancement of shear vorticity from convergence at the outflow edge allows for the production of these very strong coherent vortices that appear to be important in some cases of TCG. In the next section we discuss the presence of vortex mergers in the simulation, which help LCVAs maintain their strength.

\section{Vortex merger analysis}

Many theoretical studies have found that vortices of like sign often interact with each other and may sometimes exchange vorticity without combining altogether (e.g., Melander et al. 1987, 1988; Dritschel and Waugh 1992). Vortices have also been shown to undergo mergers during numerical simulations of TCG (Hendricks et al. 2004; Montgomery et al. 2006; Wang et al. 2010; 

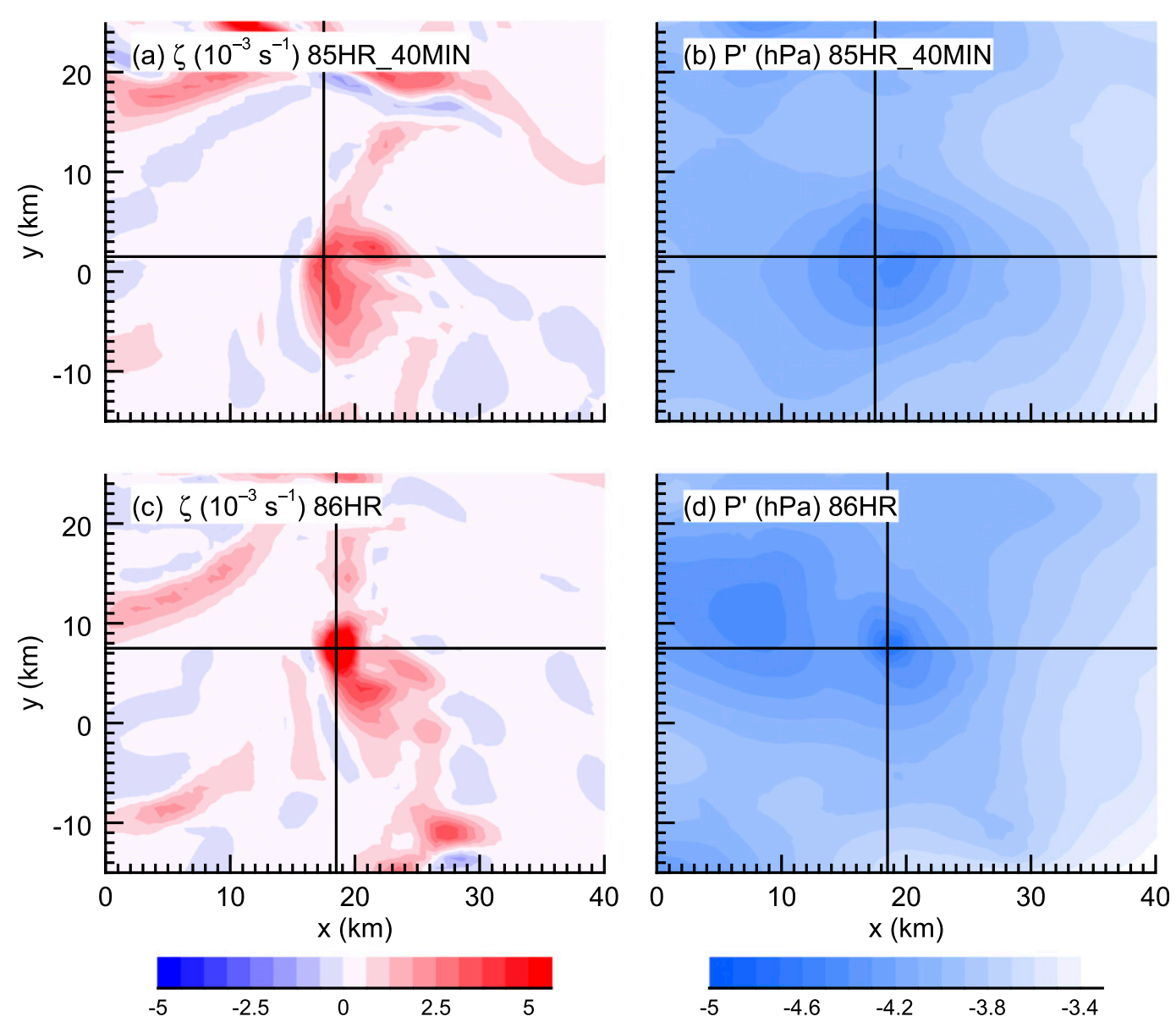

FIG. 8. Horizontal cross sections of (left) vertical vorticity and (right) perturbation pressure taken at the lowest model level ( $z=29.5 \mathrm{~m} \mathrm{AGL}$ ) at a model run time of (a),(b) $85 \mathrm{~h} 40 \mathrm{~min}$ and (c),(d) $86 \mathrm{~h}$. The crosshairs in each plot mark the center of the genesis LCVA and are included only for reference. Vorticity fields are scaled by a factor of $10^{3} \mathrm{~s}^{-1}$.

Kilroy and Smith 2013). In this section we quantitatively examine the early and genesis LCVAs for the presence of vortex mergers that may serve as a mechanism by which they can gain longevity.

Careful inspection of Fig. $4 \mathrm{c}$ reveals that the early LCVA appears to be "connected" to one of the stronger LCVAs at the point $(24,12) \mathrm{km}$ to the northeast. The vorticity to the northeast is shallow and forms at the edge of a cold pool (Fig. 4d), just as the early LCVA did in its beginning stages (Fig. 2). This same phenomenon can be observed in Fig. 9c, where the genesis LCVA appears to be "connected" to another LCVA about $20 \mathrm{~km}$ due east of it. It is hypothesized that these represent vortex merger events in which like-signed regions of vorticity can exchange their vorticity, or combine altogether. In this section we quantitatively address this claim. Specifically, it is shown that LCVAs gather lowlevel vorticity horizontally from their surroundings, which may allow them a longer lifetime as the simulation progresses and there are more numerous regions of enhanced vorticity.

To test our hypothesis quantitatively, we consider the mean integral tangential momentum budget in cylindrical coordinates utilized in Hendricks et al. [2004, their Eq. (7)]:

$$
\begin{aligned}
\bar{v}\left(t_{b}\right)-\bar{v}\left(t_{a}\right)= & -\int_{t_{a}}^{t_{b}}(\bar{u} \bar{\eta}) d t-\int_{t_{a}}^{t_{b}}\left(\bar{w} \frac{\partial \bar{v}}{\partial z}\right) d t \\
& -\int_{t_{a}}^{t_{b}}\left(\overline{u^{\prime} \zeta^{\prime}}\right) d t-\int_{t_{a}}^{t_{b}}\left(\overline{w^{\prime} \frac{\partial v^{\prime}}{\partial z}}\right) d t \\
& +\int_{t_{a}}^{t_{b}}(\overline{\mathrm{PBL}}+\overline{\mathrm{DIFF}}) d t,
\end{aligned}
$$

where an overbar indicates an azimuthal average about a defined center point, and a prime indicates the perturbation from this azimuthal mean. Put mathematically for some parameter $A$, 

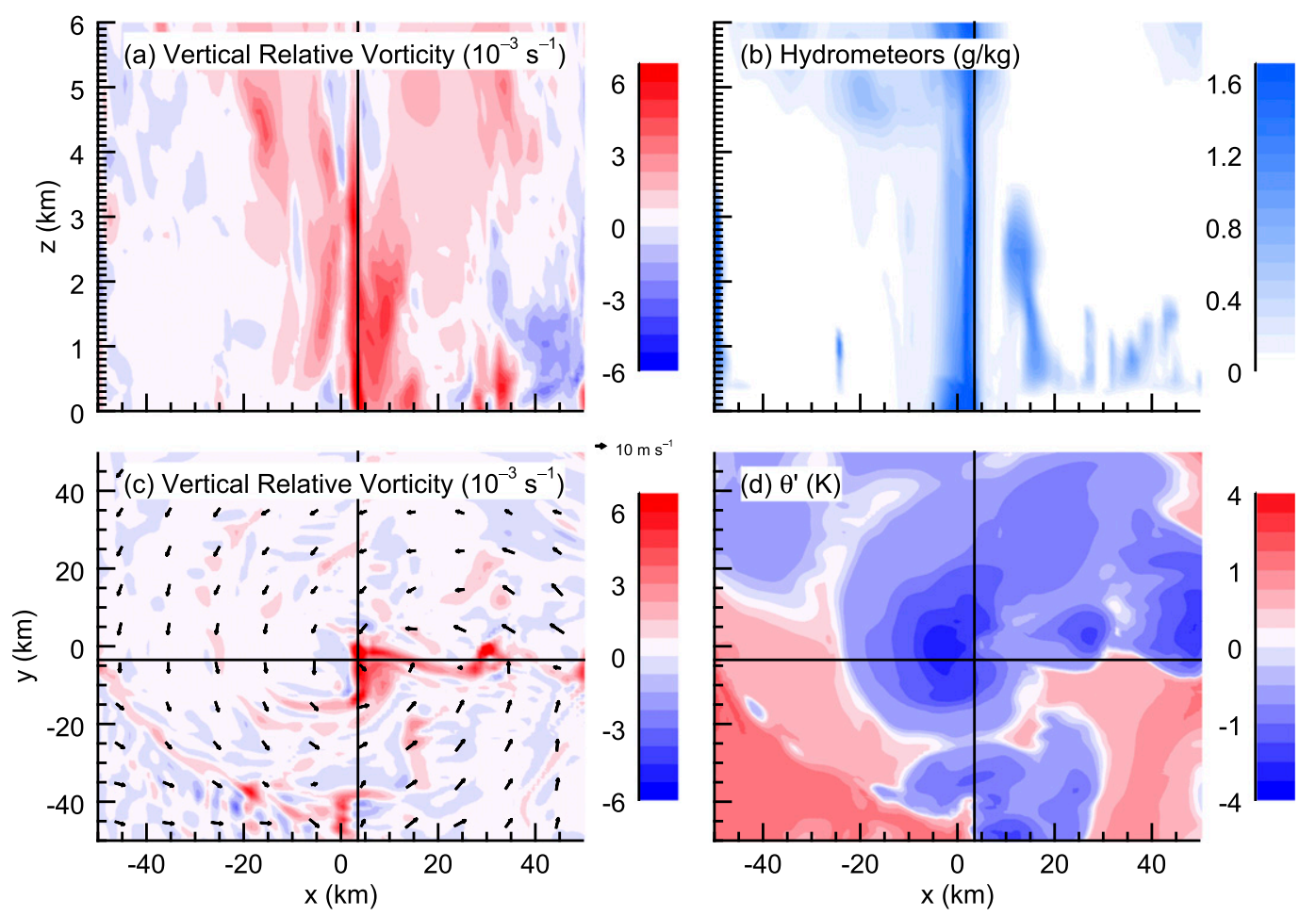

FIG. 9. As in Fig. 3, but at a model run time of $90 \mathrm{~h}$ as TCG occurs.

$$
A(r, \lambda, z, t)=\bar{A}(r, z, t)+A^{\prime}(r, \lambda, z, t) .
$$

The terms on the right-hand side of Eq. (5), from left to right, are the mean horizontal vorticity flux, mean vertical momentum flux, eddy horizontal vorticity flux, eddy vertical momentum flux, and finally the influence from the planetary boundary layer (PBL) scheme and horizontal model diffusion (DIFF). Neither PBL nor DIFF are considered in the forthcoming analysis. A positive eddy horizontal vorticity flux term is consistent with a vortex merger event (i.e., $-\overline{u^{\prime} \zeta^{\prime}} \geq 0$; Hendricks et al. 2004). Physically this means that there are coincident regions of radial inflow and positive vorticity that are strong enough to dominate in an azimuthally averaged framework, meaning that the mean tendency is for positive vorticity to propagate toward the chosen center of the coordinate system.

For azimuthal average calculations, $\Delta \lambda$ is chosen as $6.28 \times 10^{-2} \mathrm{rad}\left(\right.$ or $3.6^{\circ}$ ), so that there are 100 points in a full circular revolution. Time integrations are performed with the previous, current, and future values at each $(r, z)$ point, which in total spans a time period of $2 \mathrm{~min}$. Center points for the azimuthal averages are manually chosen as a fixed point where the smoothed maximum vorticity is found as a merger appears to be occurring. We manually altered the center point slightly and found that this does not have a large effect on these terms or the forthcoming arguments.
Figure 12 shows terms from Eq. (5) at a model run time of $48 \mathrm{~h}$, during the life cycle of the early LCVA (discussed in section 3a). At this time, the early LCVA is "joined" to another region of enhanced vorticity to its southeast. The vortex to the south is shallow, with depth on the order of hundreds of meters, as it is created from the near-surface cold pool outflow of a VHT (not shown), in similar fashion to the early stages of both LCVAs. The mean radial wind computed in the LCVAcentered coordinate system is negative (indicating inflow, not shown), which is consistent with the positive values in the mean horizontal vorticity flux (Fig. 12g) computed at this time. Near the surface and extending past $15 \mathrm{~km}$ in radius, the eddy vorticity flux (Fig. 12d) is positive and therefore consistent with a vortex merger taking place. Figures $12 \mathrm{f}$ and $12 \mathrm{i}$ [the left and right sides of Eq. (3)] are very similar to one another indicating that this budget is accurately calculated. Minor discrepancies between these panels are likely due to the PBL and DIFF terms from Eq. (5) not being included in Fig. 12i.

We now repeat this analysis for the genesis LCVA discussed in section 3b. Figure 13 shows the same fields as Fig. 12, except at a model run time of $86 \mathrm{~h} 42 \mathrm{~min}$ during the life cycle of the genesis LCVA. Figure 13a shows the genesis LCVA that appears to be "connected" to isolated regions of vorticity to the east and southeast. The positive values from the eddy horizontal vorticity 

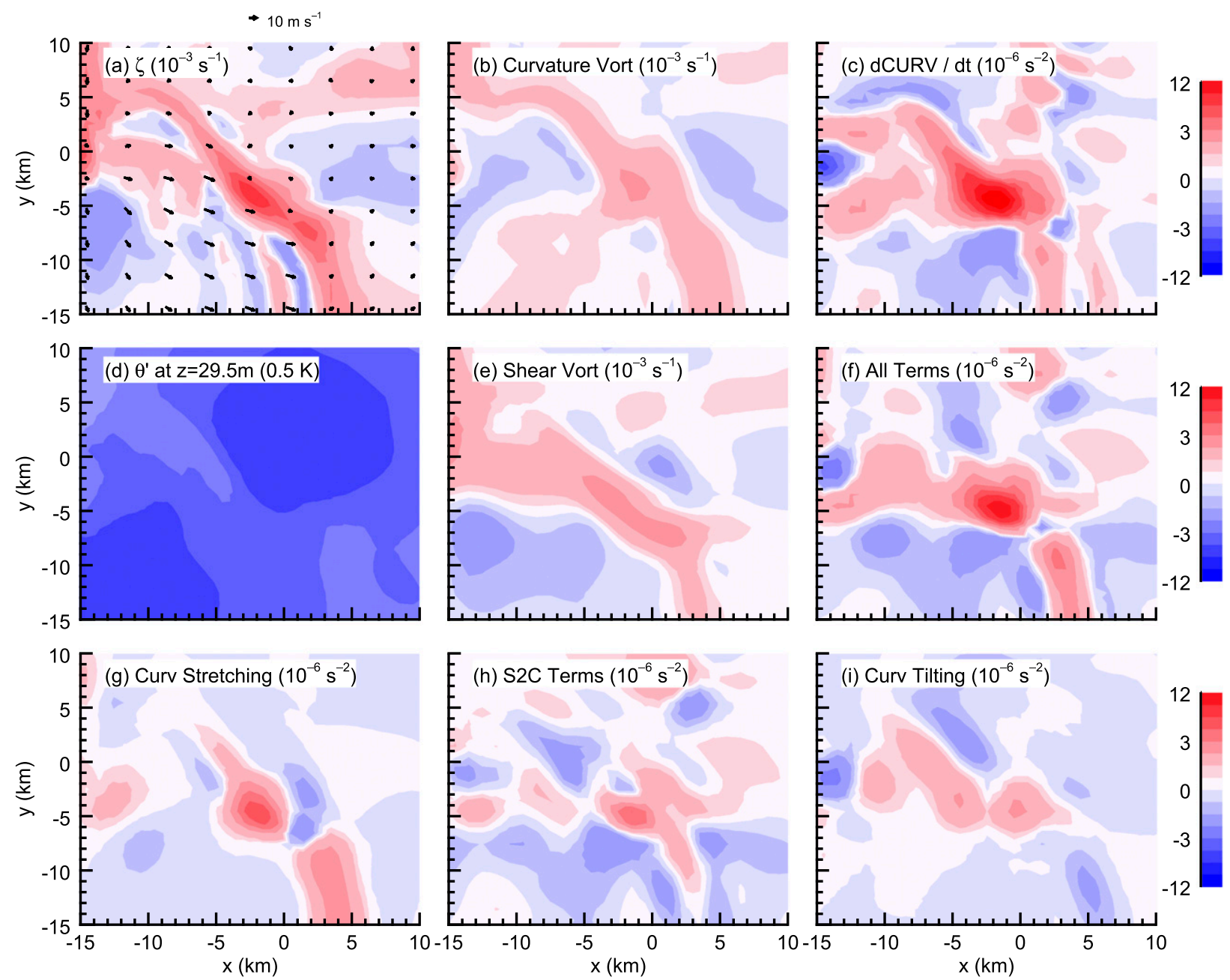

FIG. 10. Horizontal cross sections taken at $z=91.7 \mathrm{~m} \mathrm{AGL}$ at $83 \mathrm{~h} 22 \mathrm{~min}$ of (a) vertical vorticity with horizontal wind vectors, (b) curvature vorticity, (c) change in curvature vorticity in time, (d) perturbation potential temperature, (e) shear vorticity, and the (g) curvature vorticity stretching term, (h) both shear to curvature vorticity conversion terms, and (i) curvature vorticity tilting term from Eq. (4). (f) The sum of (g)-(i) and the friction term (see appendix C) for comparison with (c). All panels except (a) and (d) are smoothed once with a 1-2-1 filter. Vorticity fields are scaled by a factor of $10^{3} \mathrm{~s}^{-1}$, Eq. (4) terms are scaled by a factor of $10^{6} \mathrm{~s}^{-2}$, and the perturbation potential temperature field (which is instead taken at $z=29.5 \mathrm{~m} \mathrm{AGL}$ ) is scaled by a factor of 2 so that it fits on the same color bar as the other fields.

flux term found near the center of the LCVA are again consistent with a vortex merger event taking place (Fig. 13d). Figure 13g again indicates that radial inflow is present at low levels relative to the center of the LCVA, and comparing Figs. $13 \mathrm{f}$ and $13 \mathrm{i}$ indicates that the equation is still closed reasonably well. As the simulation progresses, additional convection provides more concentrated sources of positive low-level vorticity at the edges of convective cold pools, suggesting that LCVAs can be sustained by this mechanism for even longer as time goes on.

Based on the results from calculating this budget, it can be concluded that LCVAs draw some strength from other independently formed vorticity sources in the domain, which is consistent with previous studies. As the simulation progresses, it is possible that additional convection at smaller radii due to the contracting MLV allows for LCVAs to have an easier path to the center of the larger-scale circulation by allowing for more vortex merger events to take place at decreasing radii.

\section{Discussion}

Our findings are consistent with those presented by Fang and Zhang (2011), who noted cyclonically propagating LCVAs in their WRF simulation of Hurricane Dolly (2008), which undergo cycles of convection and mergers (section 5) causing them to persist much longer 

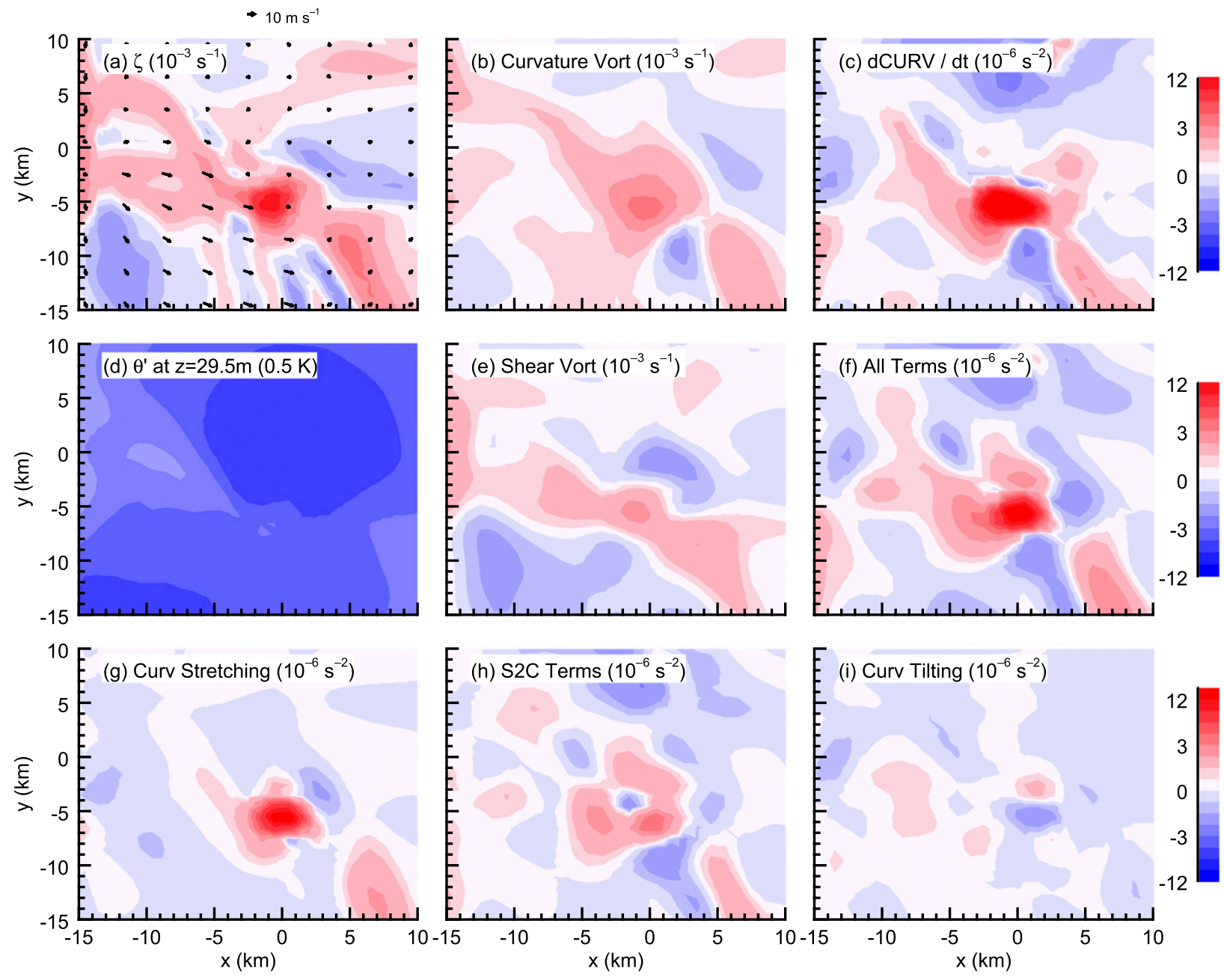

FIG. 11. As in Fig. 10, but at a model run time of $83 \mathrm{~h} 34 \mathrm{~min}$.

than a single convective tower (such as a VHT). Other numerical modeling approaches have also noted these phenomena, finding that vorticity is stretched into columns by deep convection in the development of TCs and that vortices tend to outlive the convection that forms them (Montgomery et al. 2010; Kilroy and Smith 2013).

It is worth noting that although TCG begins once the genesis LCVA reaches the center, the arrival of this low-level vortex in the center does not explain how the circulation increases in the mid- and upper troposphere during TCG because vorticity is incapable of being exchanged between isobaric surfaces, according to the arguments of Haynes and McIntyre (1987). Our primary focus is not to understand how LCVAs may promote TCG in a deep layer, but rather to understand the origin of the vortex that arrives in the center [see Figs. $3 \mathrm{~d}$ and $8 \mathrm{~d}$ in Nolan (2007)]. However, we do speculate that the presence of a strong, pronounced MLV in this simulation and the sudden convective burst that occurs as the genesis LCVA reaches the center are likely to assist with the concentration of vorticity in the mid- to upper levels as TCG begins.

The processes we explore in this study could have relevance for understanding the TCG for small TCs in weak ambient vertical wind shear. While this pathway has yet to be definitively observed in nature, a study by Sippel et al. (2006) of the genesis of Tropical Storm Allison (2001) identified several meso- $\gamma(1-10 \mathrm{~km})$ and meso- $\beta(10-100 \mathrm{~km})$-scale vortices that were considered to be playing a significant role. The observed meso- $\gamma$ vortices had RMW values of approximately $2 \mathrm{~km}$ and wind speeds on the order of $10 \mathrm{~m} \mathrm{~s}^{-1}$, consistent with our observations from the early and genesis LCVAs in our RAMS simulation. The Center for Severe Weather Research (CSWR) also observed mesoscale vortices in Hurricane Harvey (2017) as it made landfall on the coast of Texas that had comparable diameters (2$11 \mathrm{~km}$ ) to our LCVAs (Wurman and Kosiba 2018). These observational studies suggest that LCVAs may be 

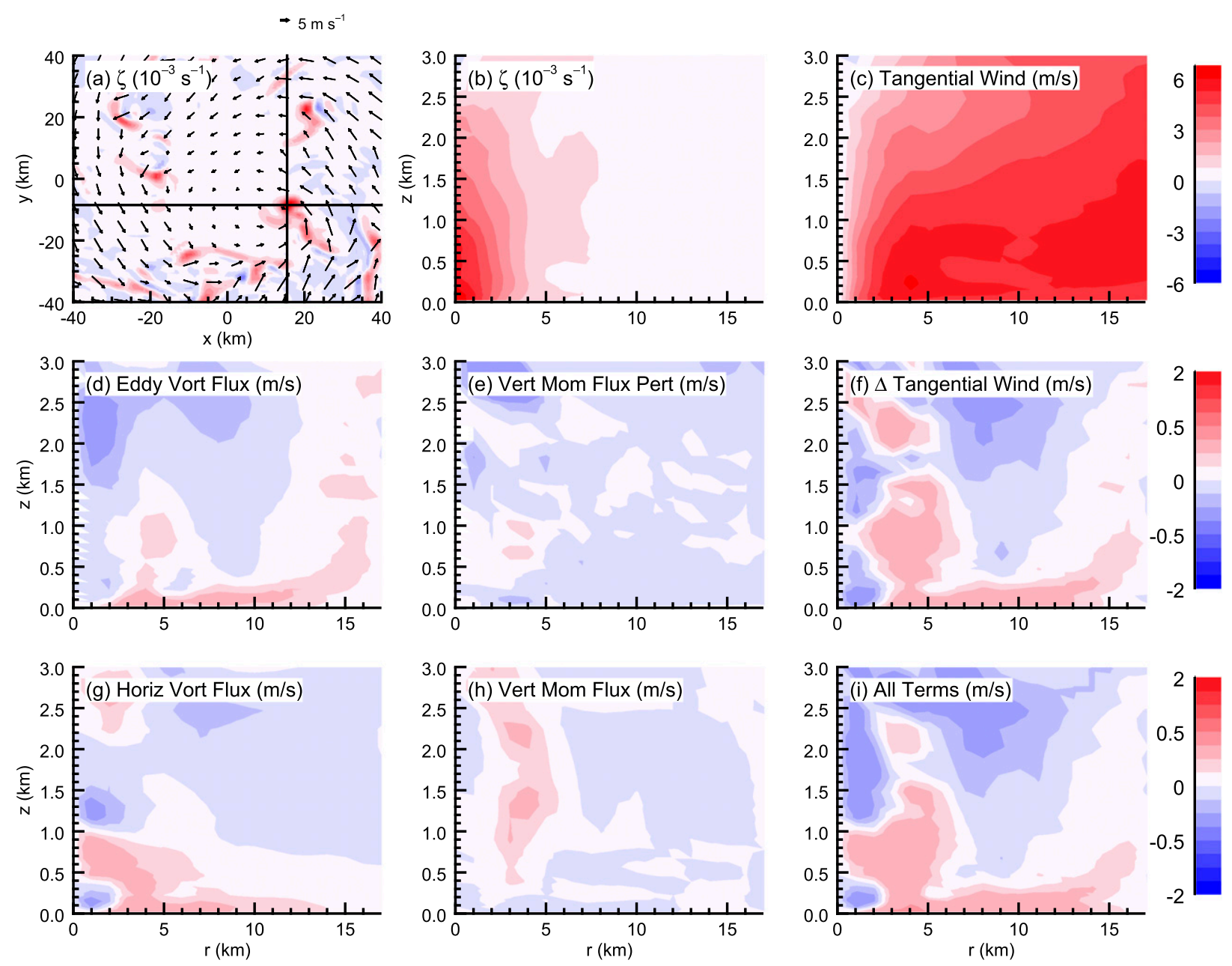

FIG. 12. Cross sections taken at a model run time of $48 \mathrm{~h}$. Shown are (a) a horizontal cross section of vertical relative vorticity with wind vectors taken at the lowest model level $(z=29.5 \mathrm{~m} \mathrm{AGL})$ and azimuthally averaged fields of (b) vertical relative vorticity, (c) tangential wind, and the (d) horizontal vorticity flux perturbation term, (e) vertical momentum flux perturbation term, (f) change in tangential wind, (g) mean horizontal vorticity flux term, and (h) vertical momentum flux term from Eq. (5). (i) The sum of panels (d), (e), (g), and (h), which comprises the right-hand side of Eq. (5) for comparison with (f). The center for all averages is marked by the crosshairs in (a). Vorticity fields are scaled by a factor of $10^{3} \mathrm{~s}^{-1}$.

important beyond the scope of TCG and may lead to enhanced damage paths as hurricanes make landfall.

The pressure drop that follows the genesis LCVA during its life cycle (Fig. 8) also reaches the domain center at $90 \mathrm{~h}$ and continues to get stronger as time progresses from Fig. 9. Since LCVAs are in approximate cyclostrophic balance (see appendix A), a pressure drop in the center of a vortex that intensifies in synchrony with the magnitude of the wind is expected. A local pressure fall with similar intensity forming over small vortices was also discussed in Nicholls and Montgomery (2013). The authors examined the formation of such small vortices when making a decision on classifying the TCG mechanism in their simulations.

In the first of a three-part study on nonsupercell tornadogenesis, Lee and Wilhelmsen modeled the creation of vortices in a similar scenario as observed here (Lee and Wilhelmson 1997). Their experimental design included an eastward-advancing cold reservoir interacting with a southerly flow. As these regions interacted, several discrete vortices developed along the interface, which marks a shear to curvature transition without the aid of convection. This result lends further support to the ability for cold pool outflows to form an LCVA from primarily shear vorticity (section 4).

\section{Conclusions}

We examine two representative low-level convectively induced vorticity anomalies (LCVAs) in an idealized RAMS simulation of tropical cyclogenesis (TCG) in an effort to understand the origin and evolution of the small 

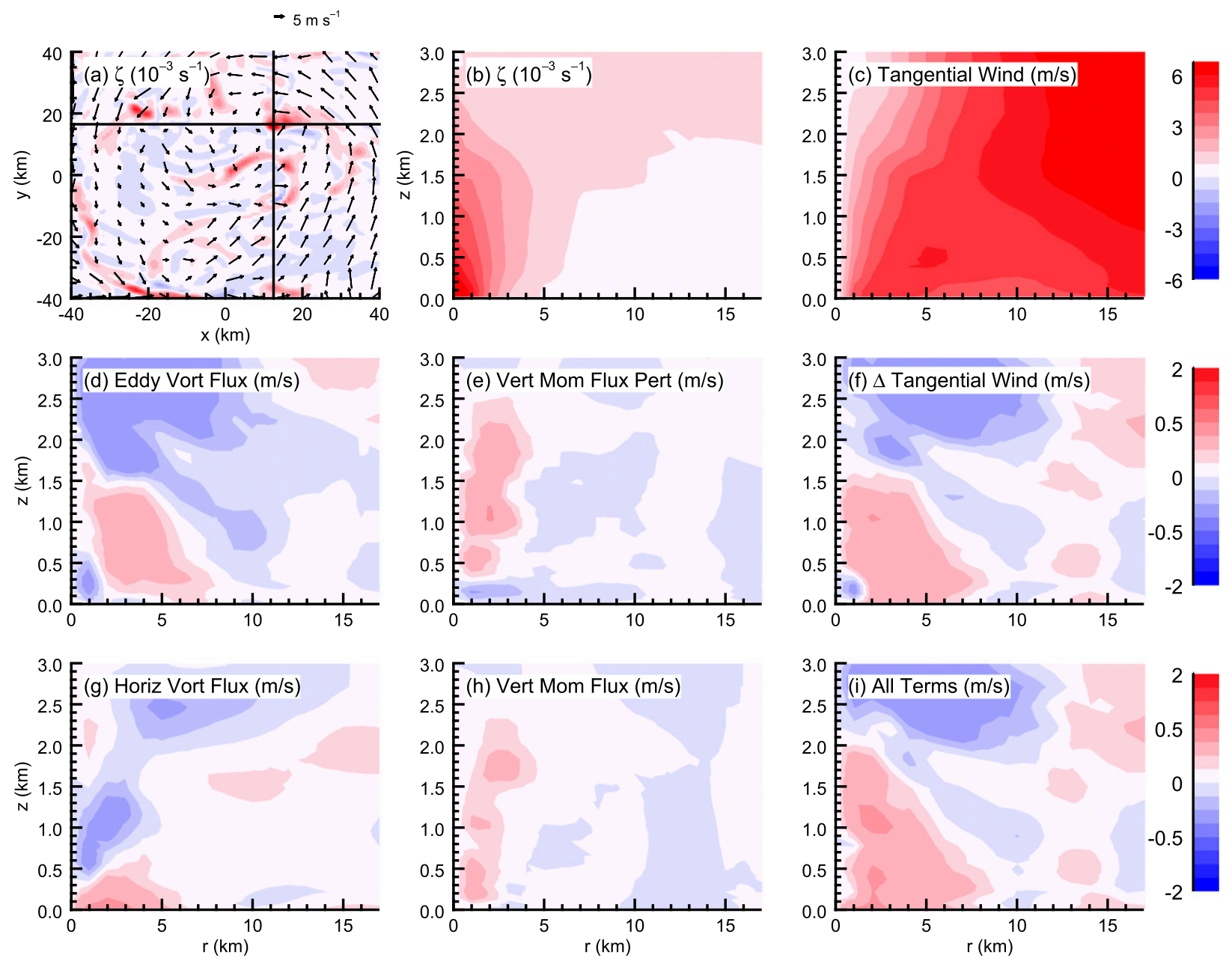

FIG. 13. As in Fig. 12, but at a model run time of $86 \mathrm{~h} 42 \mathrm{~min}$ for the genesis LCVA.

vortex (SSCV) that may form in the center of the parent circulation as TCG occurs. In our simulation it is found that LCVAs form as vorticity is concentrated by convection above regions of enhanced vorticity at the edges of cold pools. This process stretches ambient vorticity into a coherent vortex. LCVAs have maximum vorticity near the surface and outlive the convection that initially forms them. Convection occasionally strengthens these features as they cycle about the larger-scale vortex. One such LCVA that we analyze (which we termed the "early LCVA") dissipates about $8 \mathrm{~h}$ after it forms, while the other LCVA we analyze (which we termed the "genesis LCVA") is sustained until it reaches the center, where it becomes the low-level core of an intensifying TC. We confirm the ability for shear vorticity to convert to curvature vorticity at the edges of convective outflows, aiding in our understanding of how LCVAs are created. We also quantitatively validate that LCVAs undergo vortex merger events with other regions of enhanced vorticity in the model domain, which may provide a medium for LCVAs to retain their strength.

It is theorized that a strengthening MLV in this simulation increases the local vertical wind shear that allows for more persistent convection near the center of the ambient vortex as the simulation progresses (Fig. 7). More persistent convection and stronger winds at the surface are also responsible for creating more outflows that may interact to produce more enhanced regions of vorticity (Fig. 5). Additional regions of enhanced vorticity may serve to create additional LCVAs or facilitate more mergers with already active LCVAs (discussed in section 5). These arguments provide evidence that the changing background conditions of the tropical disturbance eventually become conducive for an LCVA to form and make its way into the center of the larger-scale circulation. We do not feel that the success of the genesis LCVA compared to the early LCVA can be attributed to just one process. Rather, our intention is to provide evidence for some processes that might be contributors. 
In all likelihood, some combination of the above processes allow for this development mechanism to come to fruition, rather than there being one "smoking gun" in this group.

Throughout the simulation we present herein, many LCVAs form near the center of the larger-scale circulation that do not become the beginning of a tropical depression vortex, suggesting that there are other mesoscale thermodynamic and kinematic conditions required before it becomes conducive for an LCVA to reach the center of the larger-scale circulation and intensify. There are several environmental differences between the formation of the early and genesis LCVAs that may promote the genesis LCVA reaching the center that are not discussed in this paper. These differences include (but are not limited to) deep moistening at the center (Nolan 2007), decreased low-level stability (Nicholls and Montgomery 2013), the development of a broad cold pool near the center with positive vorticity around it (Nicholls and Montgomery 2013), and a decrease in the RMW yielding higher equivalent potential temperature air closer to the center. Although we cannot address the importance of these in this work, it is worthwhile for those factors to be examined in forthcoming studies to determine their role in LCVA formation and longevity.

Additional work is likewise required to verify this phenomenon in other modeling frameworks and to observationally validate the evolution and motion of LCVAs in real developing disturbances, as well as their link to TCG that we suggest here. The connection between LCVAs and tornadoes that occur in landfalling TCs also warrants exploration because of the very large values of vorticity observed near the model surface in this simulation. Since radiation is not included in this simulation, additional investigation is required to determine if the diurnal cycle of convection observed in the tropics has any impact on the persistence of convection acting on LCVAs (Gray and Jacobson 1977). A forthcoming publication will further examine the formation of enhanced vorticity and shear to curvature vorticity conversion at the edges of convective outflows in a more detailed and idealized framework.

Acknowledgments. This work was supported by the National Science Foundation under Grants AGS-1415244 and AGS-1445875. We thank Drs. Roger Pielke, Sr., Greg Thompson, Katja Friedrich, and John Cassano for their helpful discussions about this work. We also thank Robinson Wallace for his help with developing the visualization code.

\section{APPENDIX A}

\section{Hydrostatic and Cyclostrophic Balance}

This appendix focuses on the nature of hydrostatic and cyclostrophic balances found in the genesis LCVA. Hydrostatic balance is approximately satisfied when

$$
\frac{1}{\rho_{0}} \frac{\partial p^{\prime}}{\partial z}-g \frac{\theta_{v}^{\prime}}{\theta_{v, 0}} \approx 0,
$$

where $p$ is pressure, $\rho$ is density, $\theta_{v}$ is virtual potential temperature, the subscript 0 denotes a "background" profile, and primes denote perturbations from said profile. This approach uses all points within a $20-\mathrm{km}$ radius from the model center (the center of the parent circulation) to create a unique background profile for each time step, which perturbations can then be calculated from.

Cyclostrophic wind can be derived from setting the Coriolis force $f=0$ in the formula for gradient wind balance, giving

$$
V_{\mathrm{cyc}}=\sqrt{-\frac{2 r^{2} \theta_{v, 0} \pi^{\prime}}{\left(r^{2}+a^{2}\right)}} .
$$

In Eq. (A2), $r$ denotes radius from the vortex center, $a$ is an approximate RMW, $\theta_{v, 0}$ is background virtual potential temperature as in Eq. (A1), and $\pi^{\prime}$ is the perturbation Exner function. The Exner function is defined as

$$
\pi=c_{p}\left(\frac{P}{P_{0}}\right)^{R_{d} / c_{p}}
$$

for specific heat of dry air at constant pressure $c_{p}$, dry gas constant $R_{d}$, pressure $P$, and reference pressure $P_{0}$. For LCVAs, we use $a=4.0 \mathrm{~km}$ and $P_{0}=10^{5} \mathrm{~Pa}$.

Figure A1 shows azimuthally averaged cross sections centered on the genesis LCVA at a model run time of $84 \mathrm{~h}$. Shown are tangential wind, vorticity, cyclostrophic wind, both sides of Eq. (A1), and a difference field between the two. At this time, the genesis LCVA extends to about $3 \mathrm{~km}$ AGL (Fig. A1b). A comparison of Figs. A1a and A1c shows some similarities, but the magnitude of the cyclostrophic wind is a bit higher than the observed tangential winds. The "nose" in Fig. A1a in the lowest several hundred meters is likely the result of boundary layer frictional convergence, discussed by Smith and Montgomery (2016), a process that is not reflected in the cyclostrophic wind formula. We conclude that the LCVA is in approximate cyclostrophic balance, and it is likely the relatively small effects of friction and the Coriolis force that lead to the slight magnitude disparity in these wind fields. We also conclude that the LCVA is 

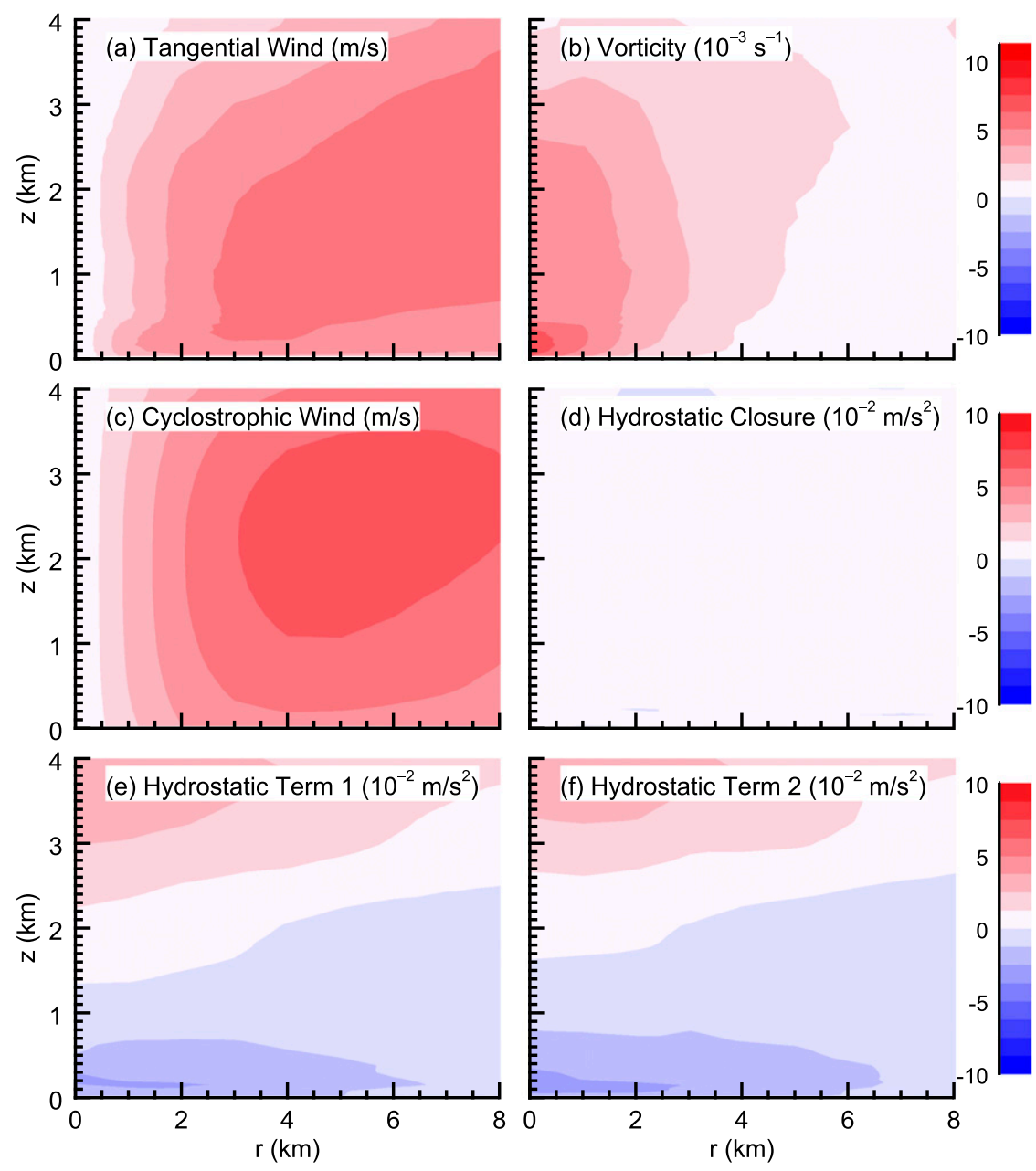

FIG. A1. Azimuthally averaged cross sections of (a) tangential wind, (b) vertical vorticity, (c) cyclostrophic wind, and the (e) first and (f) second terms in Eq. (A1) at a model run time of $84 \mathrm{~h}$ with $r=0 \mathrm{~km}$ corresponding to the center of the genesis LCVA. (d) Difference of (e) minus (f) to depict how well the hydrostatic equation closes. Vorticity is scaled by a factor of $10^{3} \mathrm{~s}^{-1}$.

in approximate hydrostatic balance at this time, evidenced by Fig. A1d having magnitudes near zero. Both of these conclusions are sensitive to the presence of convection near the LCVA; active convection causes some violation in these balances since strong vertical motion and diabatic heating are not accounted for in these simplified approximations.

\section{APPENDIX B}

\section{Surface-Based CAPE}

The importance of surface-based convective available potential energy (SBCAPE) for small, rotating vorticity anomalies has been emphasized in prior modeling studies of TCG (Fang and Zhang 2011; Nicholls and Montgomery 2013), so we feel it is important to briefly discuss its evolution in our simulation as well. Figure B1 shows horizontal cross sections of SBCAPE at various times during the evolution of the genesis LCVA. In Fig. B1a, the LCVA has not formed yet, and the enhanced region of SBCAPE serves as an important precondition for the convection that eventually forms it. After its initial formation, the SBCAPE field weakens near the LCVA (Fig. B1b) as convection briefly wanes before recovering in Fig. B1c. The fuel provided is enough to sustain convection long enough for the LCVA to reach the center (Fig. B1d). This analysis provides a window into understanding how convection is able to persist. It is theorized that the increasing surface fluxes with time allow for a faster recharge rate of SBCAPE for the genesis LCVA; however, the mechanisms responsible for this recharge warrant further investigation. 

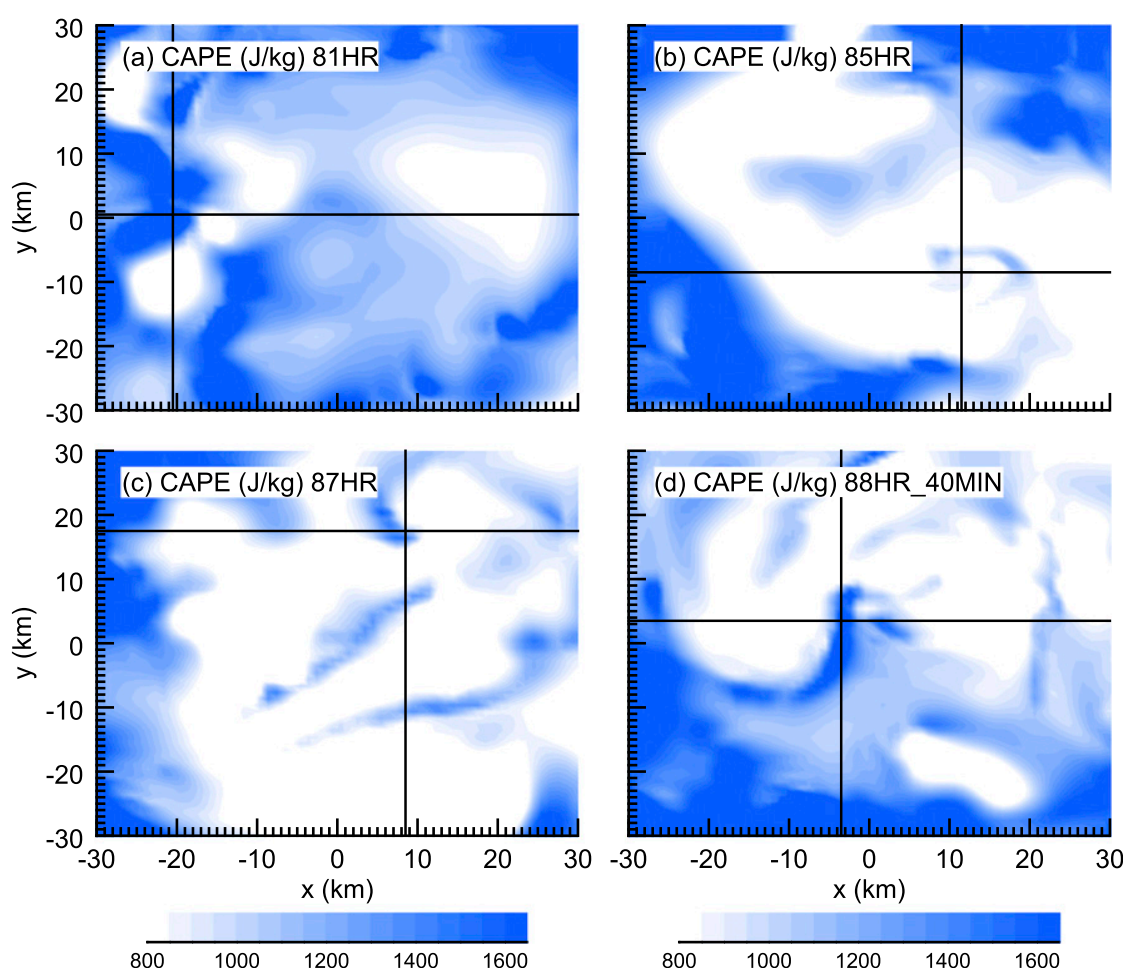

FIG. B1. Horizontal cross sections of SBCAPE at model run times of (a) $81 \mathrm{~h}$, (b) $85 \mathrm{~h}$, (c) $87 \mathrm{~h}$, and (d) $88 \mathrm{~h} 40 \mathrm{~min}$. The crosshairs in each plot mark the low-level center of the genesis LCVA and are included only for reference.

\section{APPENDIX C}

\section{Estimation of Friction for the Curvature Vorticity Budget}

Although subgrid-scale fluxes are not output from RAMS in our simulation, we feel it is appropriate to provide an estimation for the magnitude of friction given the proximity of the curvature vorticity analysis to the model surface. The friction term included in Eq. (4) is estimated according to

$$
F_{\zeta_{c}}=-g \frac{\partial}{\partial p}\left(\frac{\partial \tau_{y}}{\partial x}-\frac{\partial \tau_{x}}{\partial y}\right)
$$

where $g=9.81 \mathrm{~m} \mathrm{~s}^{-2}$ is acceleration due to gravity. The wind stresses at the surface are calculated by

$$
\begin{aligned}
\tau_{x} & =\rho C_{d} \frac{p}{p_{0}} u_{s} V_{s}, \\
\tau_{y} & =\rho C_{d} \frac{p}{p_{0}} v_{s} V_{s},
\end{aligned}
$$

where $\rho$ is the density of air; $p$ is the pressure; $p_{0}$ is a reference pressure; $u_{s}, v_{s}$, and $V_{s}$ are the surface zonal, meridional, and total wind speeds, respectively; and
$C_{d}$ is surface drag calculated by Deacon's formula (Riemer et al. 2013):

$$
C_{d}=1.1 \times 10^{-3}+\left(4.0 \times 10^{-5}\right) V_{s} .
$$

Wind stresses in Eq. (C2) are assumed to reduce to $1 \%$ of their surface value at $800 \mathrm{hPa}$ as done in Schenkel (2009).

\section{REFERENCES}

Arakawa, A., and V. R. Lamb, 1977: Computational design of the basic dynamical processes of the UCLA general circulation model. Methods in Computational Physics, J. Chang, Ed., Vol. 17, Academic Press, 173-265.

Bell, G. D., and D. Keyser, 1993: Shear and curvature vorticity and potential-vorticity interchanges: Interpretation and application to a cutoff cyclone event. Mon. Wea. Rev., 121, 76-102, https:// doi.org/10.1175/1520-0493(1993)121<0076:SACVAP > 2.0.CO;2.

Bister, M., and K. A. Emanuel, 1997: The genesis of Hurricane Guillermo: TEXMEX analyses and a modeling study. Mon. Wea. Rev., 125, 2662-2682, https://doi.org/10.1175/15200493(1997) $125<2662$ :TGOHGT > 2.0.CO;2.

Cotton, W. R., and Coauthors, 2003: RAMS 2001: Current status and future directions. Meteor. Atmos. Phys., 82, 5-29, https:// doi.org/10.1007/s00703-001-0584-9.

Davis, C. A., 2015: The formation of moist vortices and tropical cyclones in idealized simulations. J. Atmos. Sci., 72, 34993516, https://doi.org/10.1175/JAS-D-15-0027.1. 
— , and D. A. Ahijevych, 2012: Mesoscale structural evolution of three tropical weather systems observed during PREDICT. J. Atmos. Sci., 69, 1284-1305, https://doi.org/10.1175/JAS-D11-0225.1.

Drager, A. J., and S. C. van den Heever, 2017: Characterizing convective cold pools. J. Adv. Model. Earth Syst., 9, 10911115, https://doi.org/10.1002/2016MS000788.

Dritschel, D., and D. Waugh, 1992: Quantification of the inelastic interaction of unequal vortices in two-dimensional vortex dynamics. Phys. Fluids, 4A, 1737-1744, https://doi.org/10.1063/ 1.858394.

Dunkerton, T. J., M. Montgomery, and Z. Wang, 2009: Tropical cyclogenesis in a tropical wave critical layer: Easterly waves. Atmos. Chem. Phys., 9, 5587-5646, https://doi.org/10.5194/ acp-9-5587-2009.

Eastin, M. D., T. L. Gardner, M. C. Link, and K. C. Smith, 2012: Surface cold pools in the outer rainbands of Tropical Storm Hanna (2008) near landfall. Mon. Wea. Rev., 140, 471-491, https://doi.org/10.1175/MWR-D-11-00099.1.

Fang, J., and F. Zhang, 2011: Evolution of multiscale vortices in the development of Hurricane Dolly (2008). J. Atmos. Sci., 68, 103-122, https://doi.org/10.1175/2010JAS3522.1.

Gray, W. M., 1968: Global view of the origin of tropical disturbances and storms. Mon. Wea. Rev., 96, 669-700, https:// doi.org/10.1175/1520-0493(1968)096<0669:GVOTOO > 2.0.CO;2.

—, and R. W. Jacobson Jr., 1977: Diurnal variation of deep cumulus convection. Mon. Wea. Rev., 105, 1171-1188, https:// doi.org/10.1175/1520-0493(1977)105<1171:DVODCC> 2.0. $\mathrm{CO} ; 2$.

Haynes, P., and M. McIntyre, 1987: On the evolution of vorticity and potential vorticity in the presence of diabatic heating and frictional or other forces. J. Atmos. Sci., 44, 828-841, https:// doi.org/10.1175/1520-0469(1987)044<0828:OTEOVA > 2.0. $\mathrm{CO} ; 2$.

Hendricks, E. A., M. T. Montgomery, and C. A. Davis, 2004: The role of "vertical" hot towers in the formation of Tropical Cyclone Diana (1984). J. Atmos. Sci., 61, 1209-1232, https:// doi.org/10.1175/1520-0469(2004)061<1209:TROVHT> 2.0. $\mathrm{CO} ; 2$.

Hill, G. E., 1974: Factors controlling the size and spacing of cumulus clouds as revealed by numerical experiments. J. Atmos. Sci., 31, 646-673, https://doi.org/10.1175/1520-0469(1974) $031<0646$ :FCTSAS $>2.0$. CO 2 .

Holton, J. R., 1992: An Introduction to Dynamic Meteorology. 3rd ed. Academic Press, 511 pp.

Jordan, C. L., 1958: Mean soundings for the West Indies area. J. Meteor., 15, 91-97, https://doi.org/10.1175/1520-0469(1958) 015<0091:MSFTWI $>2.0 . \mathrm{CO} ; 2$.

Kilroy, G., and R. K. Smith, 2013: A numerical study of rotating convection during tropical cyclogenesis. Quart. J. Roy. Meteor. Soc., 139, 1255-1269, https://doi.org/10.1002/qj.2022.

Kutty, G., and K. Gohil, 2017: The role of mid-level vortex in the intensification and weakening of tropical cyclones. J. Earth Syst. Sci., 126, 94, https://doi.org/10.1007/s12040-017-0879-y.

Lee, B. D., and R. B. Wilhelmson, 1997: The numerical simulation of non-supercell tornadogenesis. Part I: Initiation and evolution of pretornadic misocyclone circulations along a dry outflow boundary. J. Atmos. Sci., 54, 32-60, https://doi.org/10.1175/ 1520-0469(1997)054<0032:TNSONS $>2.0$.CO;2.

Lilly, D. K., 1962: On the numerical simulation of buoyant convection. Tellus, 14, 148-172, https://doi.org/10.3402/ tellusa.v14i2.9537.
Melander, M., N. Zabusky, and J. McWilliams, 1987: Asymmetric vortex merger in two dimensions: Which vortex is "victorious"? Phys. Fluids, 30, 2610-2612, https://doi.org/10.1063/ 1.866103 .

,$- \ldots$, and $\_$, 1988: Symmetric vortex merger in two dimensions: Causes and conditions. J. Fluid Mech., 195, 303-340, https://doi.org/10.1017/S0022112088002435.

Meyers, M. P., R. L. Walko, J. Y. Harrington, and W. R. Cotton, 1997: New RAMS cloud microphysics parameterization. Part II: The two-moment scheme. Atmos. Res., 45, 3-39, https://doi.org/10.1016/S0169-8095(97)00018-5.

Montgomery, M. T., M. Nicholls, T. Cram, and A. Saunders, 2006: A vortical hot tower route to tropical cyclogenesis. J. Atmos. Sci., 63, 355-386, https://doi.org/10.1175/JAS3604.1.

_ , T. Dunkerton, and Z. Wang, 2010: Coarse, intermediate and high resolution numerical simulations of the transition of a tropical wave critical layer to a tropical storm. Atmos. Chem. Phys., 10, 10 803-10 827, https://doi.org/10.5194/acp-10-108032010.

Nicholls, M. E., and M. Montgomery, 2013: An examination of two pathways to tropical cyclogenesis occurring in idealized simulations with a cloud-resolving numerical model. Atmos. Chem. Phys., 13, 5999-6022, https://doi.org/10.5194/acp-135999-2013.

_ R. A. Pielke Sr., D. Wheeler, G. Carrio, and W. P. Smith, 2018: A numerical modelling investigation of the role of diabatic heating and cooling in the development of a mid-level vortex prior to tropical cyclogenesis-Part 1: The response to stratiform components of diabatic forcing. Atmos. Chem. Phys., 18, 14 393-14 416, https://doi.org/10.5194/acp-18-143932018.

Nolan, D. S., 2007: What is the trigger for tropical cyclogenesis? Aust. Meteor. Mag., 56, 241-266.

O'Brien, J. J., 1970: Alternative solutions to the classical vertical velocity problem. J. Appl. Meteor., 9, 197-203, https://doi.org/ 10.1175/1520-0450(1970)009<0197:ASTTCV>2.0.CO;2.

Pielke, R. A., Jr., and R. A. Pielke Sr., 1997: Hurricanes: Their Nature and Impacts on Society. John Wiley and Sons, $279 \mathrm{pp}$.

Pielke, R. A., Sr., and Coauthors, 1992: A comprehensive meteorological modeling system-RAMS. Meteor. Atmos. Phys., 49 , 69-91, https://doi.org/10.1007/BF01025401.

Raymond, D., and C. López Carrillo, 2011: The vorticity budget of developing Typhoon Nuri (2008). Atmos. Chem. Phys., 11, 147-163, https://doi.org/10.5194/acp-11-147-2011.

Riemer, M., M. Montgomery, and M. Nicholls, 2013: Further examination of the thermodynamic modification of the inflow layer of tropical cyclones by vertical wind shear. Atmos. Chem. Phys., 13, 327-346, https://doi.org/10.5194/acp-13-327-2013.

Saucier, W. J., 1955: Principles of Meteorological Analysis. Courier Corporation, $438 \mathrm{pp}$.

Schenkel, B. A., 2009: An examination of tropical cyclone evolution using curvature vorticity and shear vorticity. $\mathrm{Ph} . \mathrm{D}$. thesis, Florida State University, $172 \mathrm{pp}$.

Sippel, J. A., J. W. Nielsen-Gammon, and S. E. Allen, 2006: The multiple-vortex nature of tropical cyclogenesis. Mon. Wea. Rev., 134, 1796-1814, https://doi.org/10.1175/MWR3165.1.

Smagorinsky, J., 1963: General circulation experiments with the primitive equations: I. The basic experiment. Mon. Wea. Rev., 91, 99-164, https://doi.org/10.1175/1520-0493(1963)091<0099: GCEWTP $>2.3 . \mathrm{CO} ; 2$.

Smith, R. K., and M. T. Montgomery, 2016: Understanding hurricanes. Weather, 71, 219-223, https://doi.org/10.1002/wea.2776. 
Tripoli, G. J., and W. R. Cotton, 1981: The use of ice-liquid water potential temperature as a thermodynamic variable in deep atmospheric models. Mon. Wea. Rev., 109, 1094-1102, https://doi.org/ 10.1175/1520-0493(1981)109<1094:TUOLLW>2.0.CO;2.

Walko, R. L., W. R. Cotton, M. Meyers, and J. Harrington, 1995: New RAMS cloud microphysics parameterization-Part I: The single-moment scheme. Atmos. Res., 38, 29-62, https:// doi.org/10.1016/0169-8095(94)00087-T.
Wang, Z., M. Montgomery, and T. Dunkerton, 2010: Genesis of pre-Hurricane Felix (2007). Part II: Warm core formation, precipitation evolution, and predictability. J. Atmos. Sci., 67, 1730-1744, https://doi.org/10.1175/2010JAS3435.1.

Wurman, J., and K. Kosiba, 2018: The role of small-scale vortices in enhancing surface winds and damage in Hurricane Harvey (2017). Mon. Wea. Rev., 146, 713-722, https://doi.org/10.1175/ MWR-D-17-0327.1. 